\title{
THE PTI CARBON STAR ANGULAR SIZE SURVEY: EFFECTIVE TEMPERATURES AND NON-SPHERICITY
}

\author{
Gerard T. van Belle ${ }^{1}$, Claudia Paladini ${ }^{2}$, Bernhard Aringer ${ }^{3}$, Josef Hron $^{2}$, and David Ciardi ${ }^{4}$ \\ ${ }^{1}$ Lowell Observatory, 1400 W. Mars Hill Rd., Flagstaff, AZ, USA; gerard@lowell.edu \\ 2 Institut für Astronomie, Türkenschanzstr. 17, A-1180 Vienna, Austria; claudia.paladini@univie.ac.at, josef.hron@univie.ac.at \\ ${ }^{3}$ INAF-OAPD, Vicolo dell'Osservatorio 5, I-35122 Padova, Italy; bernhard.aringer@ oapd.inaf.it \\ ${ }^{4}$ California Institute of Technology, 770 South Wilson Avenue, Pasadena, CA, USA; ciardi@ipac.caltech.edu \\ Received 2013 February 28; accepted 2013 July 23; published 2013 September 3
}

\begin{abstract}
We report new interferometric angular diameter observations of 41 carbon stars observed with the Palomar Testbed Interferometer. Two of these stars are $\mathrm{CH}$ carbon stars and represent the first such measurements of this subtype. Of these, 39 have Yamashita spectral classes and are of sufficiently high quality that we can determine the dependence of effective temperature on spectral type. We find that there is a tendency for the effective temperature to increase with increasing temperature index by $\sim 120 \mathrm{~K}$ per step, starting at $T_{\mathrm{EFF}} \simeq 2500 \mathrm{~K}$ for $C 3, y$, although there is a large amount of scatter in this relationship. Overall, the median effective temperature of the carbon star sample is $2800 \pm 270 \mathrm{~K}$ and the median linear radius is $360 \pm 100 R_{\odot}$. We also find agreement, on average within $15 \mathrm{~K}$, with the $T_{\mathrm{EFF}}$ determinations of Bergeat et al. and a refinement of the carbon star angular size prediction based on $V \& K$ magnitudes is presented that is good to an rms of $12 \%$. A subsample of our stars have sufficient $\{u, v\}$ coverage to permit non-spherical modeling of their photospheres, and a general tendency for detection of statistically significant departures from sphericity with increasing interferometric signal-to-noise is seen. The implications of most-and potentially all-carbon stars being non-spherical is considered in the context of surface inhomogeneities and a rotation-mass-loss connection.
\end{abstract}

Key words: infrared: stars - instrumentation: high angular resolution - instrumentation: interferometers - stars: carbon - stars: distances - stars: fundamental parameters

Online-only material: color figures, machine-readable table

\section{INTRODUCTION}

General knowledge of the fundamental parameters of carbon stars is an important foundation for understanding the properties of these core constituents of the Galactic population of stars. This fact is particularly true given that carbon stars contribute $10 \%-50 \%$ of the carbon enrichment of the interstellar medium (Thronson et al. 1987). These "classical" carbon stars have spectra that are dominated by carbon species features such as $\mathrm{C}_{2}, \mathrm{C}_{2} \mathrm{H}_{2}, \mathrm{C}_{3}, \mathrm{CN}$, and $\mathrm{HCN}$ (Goebel et al. 1981; Joyce 1998; Lançon \& Wood 2000; Loidl et al. 2001). Evolutionarily speaking, this high carbon abundance is due to the third dredgeup phenomenon in the asymptotic giant branch (AGB) stars that become these carbon stars (Iben \& Renzini 1983; Herwig 2005), where the convective envelope penetrates the inter-shell region between the He- and H-burning shells, leaving these objects with $\mathrm{C} / \mathrm{O}>1$.

Yet, it remains true that very little directly obtained fundamental information exists on carbon stars. From Dyck et al. (1996b) and Bergeat et al. (2001), it is apparent that only roughly a few dozen objects from a wide variety of disparate sources have had their angular diameters and effective temperatures $\left(T_{\mathrm{EFF}}\right)$ directly determined through high-resolution techniques such as interferometry and lunar occultations. As such, the effective temperature scale for carbon stars has not been previously well established owing to these small number statistics from inhomogenous sources. Additionally, these measurements to date all represent diameters along a single measurement axis across these complex objects.

Early studies such as Mendoza V. \& Johnson (1965) found $T_{\mathrm{EFF}}$ estimates ranging from $2270 \mathrm{~K}$ to $5500 \mathrm{~K}$; later studies such as Cohen (1979) found a $T_{\mathrm{EFF}}$ scale that ranged from $3240 \mathrm{~K}$ to $2230 \mathrm{~K}$ as a function of Yamashita $(1972,1975 b)$ spectral class, largely confirmed by the interferometric measurements in Dyck et al. (1996b), who found a mean measured $T_{\mathrm{EFF}}$ of $3000 \pm 200 \mathrm{~K}$ for their sample. These authors also found a slight indication that $T_{\mathrm{EFF}}$ increases with Yamashita spectral type.

Early model spectra in Rowan-Robinson \& Harris (1983) indicated that the atmospheric temperatures for the earlier (later) types are around $2500 \mathrm{~K}(2000 \mathrm{~K})$; later models (Jørgensen et al. 2000; Loidl et al. 2001) appear to point to higher temperatures, with a mean around $3000 \mathrm{~K}$. A $T_{\mathrm{EFF}}$ calibration by Bergeat et al. (2001) attempted to extend this result, primarily through leveraging a preliminary set of Palomar Testbed Interferometer (PTI) results found by van Belle \& Thompson (1999). However, those results represent an incomplete sample with preliminary data reduction techniques; one of the intents of this manuscript is to present a finalized set of PTI data, both in terms of scope and processing.

Recently, we published a study of five carbon stars (Paladini et al. 2011), which combined infrared (IR) spectra with interferometric determinations of angular size to constrain the model atmospheres of Aringer et al. (2009). This study resulted in the successful reproduction of spectra for the first time between 0.9 and $4 \mu \mathrm{m}$ for these objects. $T_{\mathrm{EFF}}, M, \log (g), \mathrm{C} / \mathrm{O}$, and distances were derived for these objects. The aim of this study is to complement that earlier comprehensive work with a broader survey of a large sample of stars with interferometric angular sizes.

We note in particular that while this study does not have the comprehensive spectroscopic component of our Paladini et al. (2011) study, a number of the stars in this sample set have considerably more dense angular size data sets. These data sets 
allow us to establish that some, and potentially all, carbon stars present non-spherical photospheres.

The observations from the PTI facility are presented in Section 2, along with the specific carbon star models and a discussion of the details of the measured angular sizes for both the carbon stars and the giant "check" stars. Derived quantities are found in Section 3, including bolometric fluxes, effective temperatures, and distances. Finally, in Section 4, we present a discussion of the implications of these measurements, including comparisons with the works of Yamashita (1972, 1975b) and Bergeat et al. (2001, 2002a, 2002b), a calibration of the "reference" angular sizes for carbon stars, and a discussion of the possible origin of the non-spherical appearance of these stars.

\section{OBSERVATIONS AND DATA REDUCTION}

\subsection{Target Selection}

The primary source for targets was Yamashita's investigations of carbon stars (Yamashita 1972, 1975b), as already preliminarily examined in Dyck et al. (1996b). ${ }^{5}$ Yamashita spectral typing was considered by Keenan himself to be an improvement over the original Morgan and Keenan (MK) carbon star typing (Keenan 1994), although still with its own limitations (Keenan 1993). We supplement those sources with the most recent edition of Stephenson's General Catalog of Galactic Carbon Stars (Alksnis et al. 2001). From these sources, an observation list was culled, largely based on the rough observational constraints of $m_{R}<8$ (necessary for tip-tilt tracking of the individual interferometer telescopes), $m_{K}<3$ (for a sufficiently large angular size to be resolved by the interferometer), and $m_{K}>1$ (for a sufficiently small angular size to be fringe tracked by the interferometer). Roughly a dozen related S-type stars were also observed during this program and will be addressed in a separate publication. (An additional comprehensive source of well-typed carbon stars, Barnbaum et al. (1996), which builds on the typing improvements suggested in Keenan (1993), also matches well with the observational constraints of the PTI but, unfortunately, was not used in the target selection process.)

\subsection{Carbon Star Models}

For comparison with the interferometric observations, we utilized the hydrostatic carbon star models of Aringer et al. (2009), which describe the observed properties of such stars quite well. These models are improved relative to the earlier generation of models (Jørgensen et al. 2000; Loidl et al. 2001) based on their inclusion of atomic opacities and updated molecular opacities. A grid of models was generated, covering the following steps: 6 model effective temperature $2400 \leqslant$ $T_{\mathrm{EFF}}^{M} \leqslant 4000 \mathrm{~K}$ in steps of $100 \mathrm{~K}$, surface gravity $-1.0 \leqslant$ $\log g\left(\mathrm{~cm} \mathrm{~s}^{-2}\right) \leqslant+0.0$ in steps of $0.2 \mathrm{dex}$, with $Z / Z_{\odot}=1$, $M=2 M_{\odot}$, and $\mathrm{C} / \mathrm{O}=1.05$. Although additional steps of grid parameters were available $\left(M=1 M_{\odot}\right.$ and $\mathrm{C} / \mathrm{O}=1.10,1.40$, and 2.00), these options were not considered be well constrained by our data and were not exercised; in particular, the higher mass range is slightly favored by our earlier study (Paladini et al. 2011). A more detailed description of our approach with

\footnotetext{
5 The original Annals of the Tokyo Astronomical Observatory are not available electronically, but the Yamashita spectral types are present in the catalog of Skiff (2010).

6 The lower limit of $\log g$ varies with $T_{\mathrm{EFF}}$ and is detailed in Table 1 of Aringer et al. (2009).
}

the Aringer et al. (2009) models can be found in Paladini et al. (2009, 2011).

For each of the models considered herein, two complementary data sets were derived: (1) a synthetic spectral energy distribution (SED), covering the range from $0.45 \mu \mathrm{m}$ to $25 \mu \mathrm{m}$, for use with determination of the bolometric flux estimation (Section 3.1) and (2) a set of center-to-limb variation (CLV) brightness profiles specific to the PTI bandpasses, for use with determination of limb-darkened (LD) angular sizes (Section 2.5). Although these models are hydrostatic and not dynamic, our expectation was that these models were sufficient given the low-amplitude photometric variability seen for these stars across the peak of the SED (discussed in detail in Section 3.1) and thereby were sufficient for the determination of bolometric flux $\left(F_{\mathrm{BOL}}\right)$.

\subsection{PTI Observations}

The PTI was an $85-110 \mathrm{~m}$ ( $H$ and $K$ bands: $1.6 \mu \mathrm{m}$ and $2.2 \mu \mathrm{m}$ ) interferometer located at Palomar Observatory in San Diego County, CA, and is described in detail in Colavita et al. (1999). It had three $40 \mathrm{~cm}$ apertures used in pairwise combination for measurement of stellar fringe visibility on sources that range in angular size from 0.05 to 5.0 milliarcseconds (mas) and was able to resolve individual sources with angular diameters $(\theta)$ generally greater than 1.4 mas (e.g., van Belle et al. 1999, 2009) and, in certain cases, diameters roughly half of that (e.g., van Belle \& von Braun 2009). The three baselines of the PTI allowed diameter measurements across stellar photospheres at a variety of projection angles, allowing for detection of oblateness in those photospheres (van Belle et al. 2001). The PTI had been in nightly operation from 1997 until early 2009, with minimum downtime throughout the intervening years; in 2009 it was decommissioned and removed from the mountaintop.

The data from PTI considered herein cover the range from the beginning of 1998 (when the standardized data collection and pipeline reduction went into place) until the cessation of operations in 2009. In addition to the target stars discussed herein, appropriate calibration sources were observed as well and can be found en masse in van Belle et al. (2008).

\subsection{Visibility and Uniform Disk Angular Sizes}

The "canonical" calibration of the target star visibility $\left(V^{2}\right)$ data is performed by estimating the interferometer system visibility $\left(V_{\mathrm{SYS}}^{2}\right)$ using the calibration sources with model angular diameters and then normalizing the raw target star visibility by $V_{\text {SYS }}^{2}$ to estimate the $V^{2}$ measured by an ideal interferometer at that epoch (Mozurkewich et al. 1991; Boden et al. 1998; van Belle \& van Belle 2005). Uncertainties in the system visibility and the calibrated target visibility are inferred from internal scatter among the data in an observation using standard error-propagation calculations (Boden et al. 1999). Calibrating our point-like calibration objects against each other produced no evidence of systematics, with all objects delivering reduced $V^{2}=1$.

Visibility and uniform disk (UD) angular size $\left(\theta_{\mathrm{UD}}\right)$ are related using the first Bessel function $\left(J_{1}\right): V^{2}=\left[2 J_{1}(x) / x\right]^{2}$, where spatial frequency $x=\pi B \theta_{\mathrm{UD}} \lambda^{-1}$. We may establish UD angular sizes for the target stars observed by the interferometer since the accompanying parameters (projected telescope-to-telescope separation, or baseline, $B$, and wavelength of observation $\lambda$ ) are well characterized during the observation. Below, the UD 

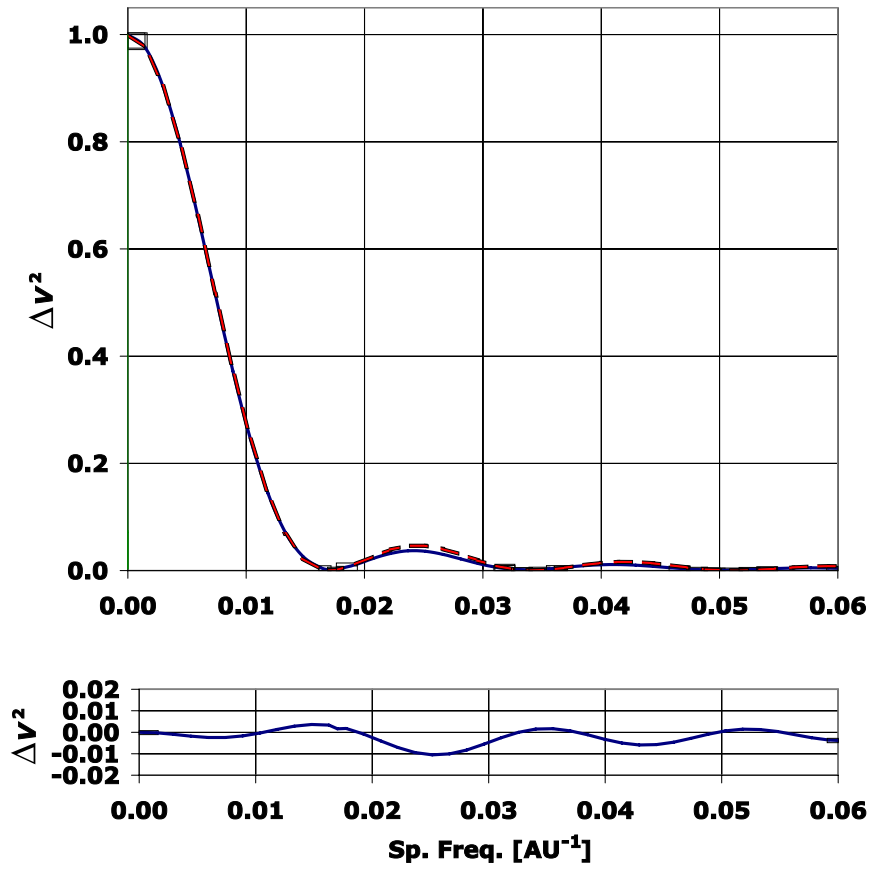

Figure 1. Representative visibility-squared curve indicated for the PTI $K$-band filter for a limb-darkened model atmosphere with $T_{\mathrm{EFF}}=2900 \mathrm{~K}$, $\log g=-0.40$, and $M=2 M_{\odot}$ (solid blue line) and a uniform disk toy model (red dashed line), based on Aringer et al. (2009). The lower panel shows the LD-to-UD residuals and has a vertical scale magnified by $5 \times$.

(A color version of this figure is available in the online journal.)

angular size will be connected to the more physical LD angular size.

\subsection{Limb-darkened Angular Sizes}

LD angular sizes $\left(\theta_{\mathrm{LD}}\right)$ are typically utilized as a reasonable proxy for the Rosseland angular diameter, which corresponds to the surface where the Rosseland mean optical depth equals unity, as advocated by Scholz \& Takeda (1987) as the most appropriate surface for computing an effective temperature. To properly consider the "true" extended atmospheres of the carbons stars observed in this investigation, we used the model CLV profiles presented in Section 2.2 that were specific to the PTI filter sets.

In general, for interferometric data taken in the central lobe (as is the case for all of our data points), the difference in visibility between LD disks and a simple UD fit is on the order of $\Delta V<1 \%$. The reason for this extremely small offset-in contrast to earlier studies that had factors of $1.022 \times$ or more (e.g., Dyck et al. 1996b) - is in the close match between the central lobes of a UD visibility curve and the LD visibility curves from the CLV brightness profiles of the Aringer et al. (2009) models (Figure 1). Comparison of the source UD CLV with the LD CLV shows that the two intersect at the $I / I(0)=\sim 50 \%$ point (Figure 2) and that both match the linear "radius" defined for these models, which fundamentally is set by the models' Rosseland radii, where $\tau_{\text {ROSS }}=2 / 3$. A net result of this definition is that, for the central lobe visibility data, there a is significant correspondence between the "toy" UD model and the visibility data from the model CLV. As such, an LD-to-UD conversion factor will be not be implemented here.

Table 1 presents our observed angular sizes, including target IDs, coordinates, the number of visibility points $N\left(V^{2}\right)$ (available and used), derived $\theta_{\text {UD }}$ sizes, associated goodness of fit as characterized by a reduced chi-squared $\left(\chi_{v}^{2}\right)$, and average squared visibility residuals.

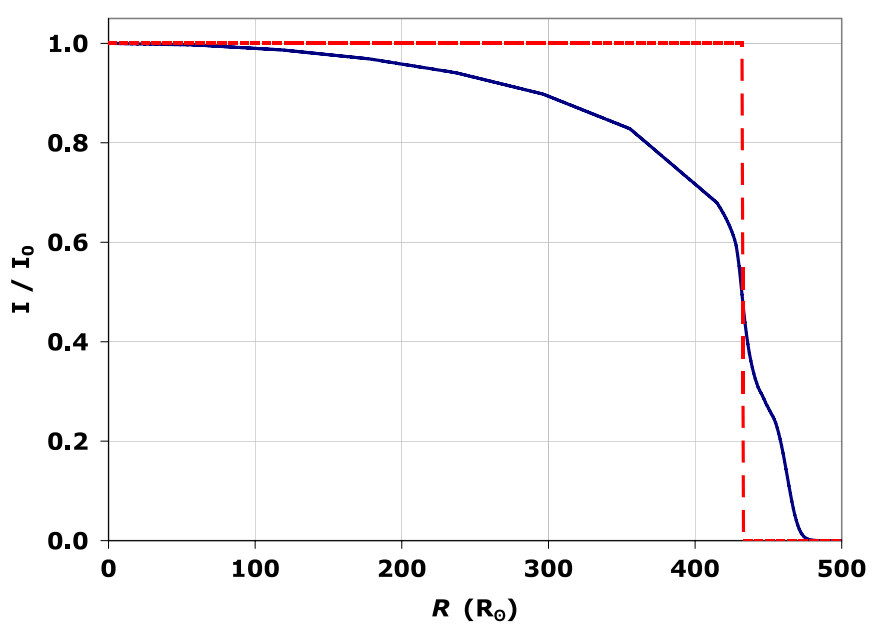

Figure 2. Plot of a representative CLV curve indicated for the PTI $K$-band filter for a limb-darkened model atmosphere with $T_{\mathrm{EFF}}=2900 \mathrm{~K}, \log g=-0.40$, and $M=2 M_{\odot}$ (solid blue line) and a uniform disk toy model (red dashed line), based on Aringer et al. (2009).

(A color version of this figure is available in the online journal.)

\subsection{Variability of Angular Sizes}

\subsubsection{Temporal}

All of these carbon stars exhibit photometric variability, particularly in the visible, where $\Delta V \simeq 1$ is not uncommon (Watson 2006). However, in the near-IR (where the bulk of the spectral energy is emitted), the variability is significantly less (Section 3.1). For HIP 92194 and HIP 113715, with $N>50$ data points over $\Delta t>400$ days, each underwent a Lomb-Scargle periodogram analysis (Lomb 1976; Scargle 1982) to see if any periodicity was detectable in the angular size data. None was detected, unlike the $\sim \pm 20 \%$ angular diameter pulsations seen for Miras (Thompson et al. 2002), which have far greater variability with $\Delta V \gtrsim 3$. Examination of the visible light curves for these objects show behavior that is markedly more stochastic than is the case for Miras.

\subsubsection{Size versus Position Angle for Carbon Stars}

Recent studies have shown that certain stars can take on a non-spherical appearance due to the effects of surface inhomogeneities (Chiavassa et al. 2011a), rotation (van Belle et al. 2001; van Belle 2012), or-of particular interest in the case of carbon stars - undergoing extreme mass loss (as illustrated for IRC+10216 in Figure 1 of Tuthill et al. 2005). For interferometry data of this nature-namely, very high resolution but not particularly well sampled with regard to full image reconstruction-other phenomena can take on the appearance of a varying UD size with changes in position angle. Specifically, a circular stellar disk with prominent, non-central surface inhomogeneities is one solution, as well as a circular stellar disk with an encircling disk. To inform our discussion of the possible sources in Section 4.5, we will first establish for which stars we can confidently measure departures from on-sky spherical symmetry.

For most of our sample, there is insufficient $\{u, v\}$ plane sampling to definitively establish for each individual object whether or not it is non-spherical. However, for 12 of our stars, we managed to obtain visibility measurements for both the north-south (NS) and north-west (NW) baselines of the PTI, permitting examination of these stars as non-spherical objects. 
Table 1

Observed Targets, Coordinates, Number of Visibility Points, Resultant Uniform Disk Angular Size, and Visible Variability Information

\begin{tabular}{|c|c|c|c|c|c|c|c|c|c|c|c|c|c|}
\hline \multirow[b]{2}{*}{ Target ID } & \multirow[b]{2}{*}{ Alt. } & \multirow[b]{2}{*}{ R.A. (J2000) } & \multirow[b]{2}{*}{ Decl. (J2000) } & \multirow[b]{2}{*}{$\begin{array}{c}V^{2} \\
\text { Points }^{\mathrm{a}}\end{array}$} & \multirow[b]{2}{*}{$\begin{array}{c}\theta^{\mathrm{b}} \\
(\mathrm{mas})\end{array}$} & \multirow[b]{2}{*}{$\chi^{2} / \mathrm{dof}$} & \multirow[b]{2}{*}{$\begin{array}{l}\text { Average } \\
\text { Residual }\end{array}$} & \multirow[b]{2}{*}{ Group $^{\mathrm{c}}$} & \multicolumn{3}{|c|}{ GCVS $^{\mathrm{d}}$} & \multirow{2}{*}{$\begin{array}{c}\text { AFOEV } \\
\text { Amp } \\
\text { (mag) }\end{array}$} & \multirow{2}{*}{$\begin{array}{l}\text { HIP } \\
\text { Amp } \\
\text { (mag) }\end{array}$} \\
\hline & & & & & & & & & Var. Type & $\begin{array}{l}\text { Amp } \\
\text { (mag) }\end{array}$ & Band & & \\
\hline HD 225217 & SU And & 000436.4 & +433304.7 & $10 / 10$ & $2.577 \pm 0.021$ & 0.855 & 0.016 & CV3 & $\mathrm{LC}$ & 0.50 & V & 0.80 & 0.23 \\
\hline HD 2342 & AQ And & 002731.6 & +353514.4 & $2 / 2$ & $3.867 \pm 0.015$ & 0.217 & 0.001 & CV5 & SR & 1.90 & $\mathrm{p}$ & 1.80 & 0.36 \\
\hline HD 19881 & V410 Per & 031338.6 & +474933.8 & $19 / 21$ & $2.357 \pm 0.011$ & 0.649 & 0.017 & $\mathrm{CV} 3$ & SRB: & 0.60 & V & 1.10 & 0.28 \\
\hline IRC+40067 & AC Per & 034503.3 & +444651.0 & $11 / 12$ & $3.281 \pm 0.018$ & 0.797 & 0.010 & CV5 & LB: & 0.60 & $\mathrm{p}$ & & \\
\hline HD 30443 & & 044916.0 & +350006.4 & $3 / 3$ & $1.430 \pm 0.094$ & 0.226 & 0.020 & $\mathrm{HC} 4$ & & & & & \\
\hline HD 280188 & V346 Aur & 045234.8 & +383019.9 & $1 / 1$ & $3.399 \pm 0.028$ & inf & & $\mathrm{SCV}$ & SRA: & 1.01 & $B$ & 1.10 & 0.26 \\
\hline HD 33016 & TX Aur & 050905.4 & +390008.4 & $3 / 3$ & $2.945 \pm 0.036$ & 0.701 & 0.012 & CV4 & LB & 0.70 & $V$ & 0.90 & 0.28 \\
\hline HD 34467 & V348 Aur & 051910.2 & +354732.4 & $2 / 2$ & $2.237 \pm 0.049$ & 0.005 & 0.001 & $\mathrm{CV} 3$ & LB & 1.05 & $B$ & 0.60 & 0.15 \\
\hline HIP 25004 & V1368 Ori & 052113.3 & +072119.3 & $2 / 2$ & $3.283 \pm 0.042$ & 0.230 & 0.005 & $\mathrm{CV} 2$ & SRA & 0.35 & $\mathrm{Hp}$ & & 0.35 \\
\hline HD 38218 & TU Tau & 054513.7 & +242512.4 & $2 / 4$ & $3.894 \pm 0.011$ & 2.050 & 0.002 & CV3 & SRB & 3.30 & V & 2.20 & 0.46 \\
\hline HD 247224 & CP Tau & 054526.5 & +153045.3 & $2 / 2$ & $1.602 \pm 0.069$ & 0.276 & 0.011 & $\mathrm{CV} 3$ & LB & 1.90 & $\mathrm{p}$ & 1.40 & \\
\hline HD 38572 & FU Aur & 054808.1 & +30 3751.8 & $2 / 2$ & $2.664 \pm 0.027$ & 0.009 & 0.001 & $\mathrm{CV} 2$ & LB & 1.20 & $B$ & 1.00 & 0.23 \\
\hline HD 38521 & AF Aur & 054844.7 & +445436.0 & $2 / 2$ & $2.331 \pm 0.046$ & 0.912 & 0.015 & & SR: & 3.20 & $\mathrm{p}$ & 1.70 & \\
\hline HIP 29896 & GK Ori & 061742.0 & +083111.3 & $5 / 5$ & $3.500 \pm 0.022$ & 0.580 & 0.008 & CV6 & SR & 1.50 & $V$ & 1.50 & 0.31 \\
\hline HD 45087 & $\mathrm{AB}$ Gem & 062614.0 & +190423.0 & $4 / 4$ & $2.755 \pm 0.031$ & 0.501 & 0.010 & CV6 & LB & 2.20 & $\mathrm{p}$ & 2.00 & \\
\hline HIP 31349 & CR Gem & 063423.9 & +160430.3 & $1 / 1$ & $3.864 \pm 0.015$ & inf & $\mathrm{N} / \mathrm{A}$ & $\mathrm{CV} 2$ & LB & 1.20 & $B$ & 1.00 & 0.54 \\
\hline HD 46321 & RV Aur & 063444.6 & +423012.7 & $4 / 4$ & $2.126 \pm 0.064$ & 2.196 & 0.044 & CV3 & SRB & 1.30 & $\mathrm{p}$ & 1.50 & 0.16 \\
\hline HD 47883 & VW Gem & 064208.5 & +312717.5 & $8 / 8$ & $2.187 \pm 0.028$ & 0.512 & & & LB & 0.38 & $V$ & & 0.23 \\
\hline HD 48664 & CZ Mon & 064440.8 & +03 1900.0 & $5 / 5$ & $2.883 \pm 0.040$ & 0.503 & 0.015 & CV5 & LB & 2.00 & $\mathrm{p}$ & & \\
\hline HD 51620 & RV Mon & 065821.4 & +061001.5 & $1 / 1$ & $3.421 \pm 0.062$ & inf & $\mathrm{N} / \mathrm{A}$ & $\mathrm{CV} 3$ & SRB & 2.19 & $B$ & 2.40 & 0.39 \\
\hline HD 54361 & W CMa & 070803.4 & -115523.7 & $7 / 7$ & $4.890 \pm 0.016$ & 0.471 & 0.003 & $\mathrm{CV} 3$ & LB & 1.55 & V & 2.00 & 0.45 \\
\hline IRC +10158 & $\mathrm{BK} \mathrm{CMi}$ & 071538.8 & +050339.7 & $6 / 6$ & $2.946 \pm 0.046$ & 0.745 & 0.033 & CV5 & & & & 1.50 & \\
\hline HD 57160 & BM Gem & 072059.0 & +245958.0 & $1 / 1$ & $2.213 \pm 0.028$ & inf & $\mathrm{N} / \mathrm{A}$ & CV1 & SRB & 0.60 & $\mathrm{p}$ & 0.93 & 0.29 \\
\hline HD 59643 & NQ Gem & 073154.5 & +243012.5 & $41 / 41$ & $1.499 \pm 0.066$ & 2.580 & 0.101 & $\mathrm{HC} 5$ & SR+ZAND & 0.59 & $V$ & 1.50 & 0.18 \\
\hline HD 60826 & BE CMi & 073629.1 & +020444.1 & $3 / 3$ & $2.396 \pm 0.070$ & 0.079 & 0.009 & CV3 & LB & 0.50 & $\mathrm{p}$ & 0.55 & \\
\hline HD 70072 & RY Hya & 082006.3 & +024556.0 & $11 / 13$ & $4.112 \pm 0.011$ & 1.854 & 0.011 & CV6 & SRB & 2.80 & $\mathrm{p}$ & 2.75 & \\
\hline HD 144578 & RR Her & 160413.4 & +502956.9 & $58 / 64$ & $1.582 \pm 0.015$ & 1.633 & 0.047 & $\mathrm{CV} 2$ & SRB & 4.70 & $B$ & 2.60 & 1.61 \\
\hline HD 173291 & HK Lyr & 184250.0 & +365730.8 & $6 / 9$ & $3.945 \pm 0.006$ & 2.924 & 0.003 & $\mathrm{CV} 3$ & LB & 1.80 & V & 1.60 & 0.32 \\
\hline HIP 92194 & DR Ser & 184721.0 & +052718.6 & $54 / 75$ & $3.447 \pm 0.005$ & 2.375 & 0.017 & CV5 & LB & 2.99 & $B$ & 0.40 & 0.27 \\
\hline HIP 95024 & U Lyr & 19200 & +375 & $5 / 7$ & $3.933 \pm 0.0$ & & & $\mathrm{CV}$ & & & & 2.80 & \\
\hline HIP 95777 & AW Cyg & 192847.5 & +460238.1 & $8 / 9$ & $3.387 \pm 0.010$ & 6.115 & 0.016 & $\mathrm{CV} 4$ & SRB & 3.50 & $\mathrm{p}$ & 2.50 & 0.29 \\
\hline HD 186047 & TT Cyg & 194057.0 & +323705.7 & $1 / 1$ & $3.305 \pm 0.024$ & inf & $\mathrm{N} / \mathrm{A}$ & $\mathrm{CV} 4$ & SRB & 1.70 & $B$ & 1.70 & 0.26 \\
\hline HIP 99336 & AY Cyg & 200944.2 & +412936.4 & $2 / 2$ & $2.156 \pm 0.128$ & 4.568 & 0.075 & CV5 & LB & 2.20 & $B$ & 0.80 & 0.32 \\
\hline HD 191783 & RY Cyg & 201023.0 & +355650.1 & $17 / 17$ & $2.382 \pm 0.017$ & 1.283 & 0.033 & CV4 & LB & 1.80 & V & 2.00 & \\
\hline HIP 102764 & V1862 Cyg & 204916.2 & +331347.1 & $17 / 20$ & $2.902 \pm 0.010$ & 3.899 & 0.031 & CV6 & $\mathrm{SR}$ & 2.60 & $B$ & & 0.33 \\
\hline HIP 105539 & YY Cyg & 212228.7 & +422346.4 & $42 / 49$ & $2.576 \pm 0.007$ & 1.288 & 0.016 & $\mathrm{CV} 4$ & SRA & 1.10 & $\mathrm{p}$ & 0.80 & 0.51 \\
\hline IRC+50399 & LQ Cyg & 214639.1 & +523400.0 & $1 / 1$ & $2.069 \pm 0.098$ & inf & $\mathrm{N} / \mathrm{A}$ & & LB: & 1.10 & $B$ & & \\
\hline HD 208512 & LW Cyg & 215513.7 & +502949.7 & $2 / 2$ & $3.954 \pm 0.025$ & 1.045 & 0.003 & CV5 & LB & 2.20 & $B$ & 1.50 & 0.26 \\
\hline HD 208526 & RX Peg & 215622.3 & +225139.9 & $1 / 1$ & $2.988 \pm 0.127$ & inf & $\mathrm{N} / \mathrm{A}$ & $\mathrm{CV} 3$ & SRB & 1.90 & $\mathrm{p}$ & 1.90 & \\
\hline HIP 113715 & VY And & 230149.4 & +455309.1 & $96 / 116$ & $2.456 \pm 0.005$ & 1.758 & 0.021 & CV5 & SRB & 2.20 & $V$ & 1.40 & 0.64 \\
\hline HD 220870 & EW And & 232657.3 & +493058.9 & $71 / 73$ & $1.904 \pm 0.008$ & 1.217 & 0.023 & CV3 & LB: & 1.00 & $\mathrm{p}$ & 1.30 & 0.26 \\
\hline
\end{tabular}

Notes. No data available for HD 30443, IRC+10158, or HIP 95024. AFOEV data have been selected for the available $V$-band data.

a Visibility points used in fitting $\theta_{\mathrm{UD}}$, vs. number available; $3 \sigma$ outliers were discarded.

${ }^{b}$ Error bar is the formal fitting error. For ensuing computations that make use of $\theta_{\mathrm{UD}}$, an error floor of $\pm 4.6 \%$ will be used, as per the discussion at the end of Section 2.6.2.

${ }^{\mathrm{c}}$ From the group designations in Bergeat et al. (2001), as discussed in Section 4.2.

d Variability type and amplitude from the AAVSO Variability Index (Watson et al. 2012); amplitude indicated as $V$ band, $B$ band, photographic (p), or $\operatorname{Hipparcos}(\mathrm{Hp})$.

For each of these 12 stars, each individual visibility point was fit with a corresponding UD diameter. These UD diameters were then collectively fit with (1) a simple single-parameter circle $\left(\theta_{\text {circ }}\right)$ and (2) an ellipsoidal fit, with a major axis, minor axis, and position angle $(a, b$, and $\phi$ ) (Table 2; Figures 3 and 4$).^{7} \mathrm{~A}$ few of the visibility points are clear outliers and are discarded (as noted in the captions of Figures 3 and 4). Visibility measurements were made at a variety of baselines

\footnotetext{
7 Individual calibrated visibility points and associated metadata (observation MJD, baseline data including length, and apparent position angle) are available upon request for any star in our sample (1305 in total). All raw visibility data are also available online at the PTI archive, http://nexsci.caltech.edu.
}

corresponding to the central lobe of the star's visibility curve. Slight differences can be expected between the UD visibility function and the "true" stellar visibility curve, but, as seen in Figure 1, the differences will be at the $\ll 1 \%$ level-well below the threshold of our measurement error.

Overall, the ellipsoidal fit is an improved characterization of the angular size data; for one object (HD 59643) the data are insufficient to properly constrain the elliptical fit and for a second (HD 144578) the elliptical fit does not improve upon the circular fit. For the rest of this subset of the target sample, an elliptical fit is an improvement, although statistically significant (with a improvement in reduced chi-squared of 
Table 2

Parameters of Ellipsoidal Fits to Angular Size Data for Those Stars with Semi-orthogonal NS and NW Baseline Data

\begin{tabular}{|c|c|c|c|c|c|c|c|c|c|c|}
\hline Star ID & $\begin{array}{l}\theta_{\text {circ }} \\
\text { (mas) }\end{array}$ & $\begin{array}{c}a \\
\text { (mas) }\end{array}$ & $\begin{array}{c}b \\
\text { (mas) }\end{array}$ & $\begin{array}{c}\phi \\
\left({ }^{\circ}\right)^{\mathrm{a}}\end{array}$ & $\chi_{v, \text { ellipse }}^{2}$ & $\chi_{v, \text { circle }}^{2}$ & $\Delta \chi_{v}^{2}$ & $\begin{array}{l}\theta_{\text {equiv }} \\
\text { (mas) }\end{array}$ & $\frac{\theta_{\text {equiv }}}{\theta_{\text {circ }}}$ & $\begin{array}{c}\text { Oblateness } \\
o_{a b}=a / b-1\end{array}$ \\
\hline HD 19881 & $2.371 \pm 0.010$ & $2.441 \pm 0.085$ & $2.165 \pm 0.096$ & $-50 \pm 8$ & 1.1 & 1.6 & 0.4 & 2.298 & 0.969 & $0.128^{+0.093}-0.128$ \\
\hline IRC+40067 & $3.297 \pm 0.018$ & $3.396 \pm 0.079$ & $3.005 \pm 0.160$ & $-47 \pm 6$ & 0.8 & 1.7 & 0.8 & 3.194 & 0.969 & $0.130^{+0.087}-0.073$ \\
\hline HD 59643 & $1.499 \pm 0.024$ & 1.766 & 1.476 & 57 & 1.2 & 1.2 & 0.0 & 1.614 & 1.077 & 0.197 \\
\hline HD 144578 & $1.642 \pm 0.015$ & $1.658 \pm 0.036$ & $1.612 \pm 0.080$ & -78 & 2.2 & 2.1 & -0.1 & 1.635 & 0.995 & $0.029^{+0.096}-0.029$ \\
\hline HIP 92194 & $3.459 \pm 0.004$ & $3.917 \pm 0.086$ & $3.365 \pm 0.016$ & $28 \pm 1$ & 2.0 & 4.8 & 2.7 & 3.630 & 1.049 & $0.164^{+0.032}-0.037$ \\
\hline HIP 95024 & $3.808 \pm 0.018$ & $3.951 \pm 0.048$ & $3.588 \pm 0.053$ & $-76 \pm 12$ & 0.1 & 14.1 & 14.1 & 3.765 & 0.989 & $0.101^{+0.038}-0.030$ \\
\hline HIP 95777 & $3.394 \pm 0.010$ & $3.634 \pm 0.076$ & $3.152 \pm 0.062$ & $-54 \pm 11$ & 2.6 & 7.8 & 5.2 & 3.385 & 0.997 & $0.153^{+0.042}-0.049$ \\
\hline HD 191783 & $2.391 \pm 0.018$ & $2.432 \pm 0.048$ & $2.174 \pm 0.170$ & $-62 \pm 12$ & 1.2 & 1.5 & 0.3 & 2.300 & 0.962 & $0.118^{+0.065}-0.118$ \\
\hline HIP 102764 & $2.938 \pm 0.010$ & $3.191 \pm 0.068$ & $2.846 \pm 0.024$ & $76 \pm 7$ & 1.3 & 5.7 & 4.4 & 3.013 & 1.026 & $0.121^{+0.027}-0.039$ \\
\hline HIP 105539 & $2.619 \pm 0.005$ & $2.776 \pm 0.047$ & $2.539 \pm 0.022$ & $42 \pm 1$ & 2.2 & 3.2 & 1.0 & 2.655 & 1.014 & $0.093^{+0.026}-0.032$ \\
\hline HIP 113715 & $2.450 \pm 0.004$ & $2.530 \pm 0.030$ & $2.429 \pm 0.010$ & $66 \pm 11$ & 3.8 & 4.0 & 0.2 & 2.479 & 1.012 & $0.042^{+0.014}-0.042$ \\
\hline HD 220870 & $1.901 \pm 0.007$ & $1.940 \pm 0.020$ & $1.839 \pm 0.027$ & $-75 \pm 18$ & 0.9 & 1.3 & 0.4 & 1.889 & 0.993 & $0.055^{+0.049}-0.055$ \\
\hline \multicolumn{11}{|c|}{ Check stars (NS, NW baselines only) } \\
\hline HD 113226 & $3.062 \pm 0.009$ & $3.073 \pm 0.096$ & $3.001 \pm 0.073$ & $-7 \pm 42$ & 2.1 & 1.9 & -0.2 & 3.037 & 0.992 & $0.024^{+0.033}-0.024$ \\
\hline HD 216131 & $2.366 \pm 0.011$ & $2.388 \pm 0.159$ & $2.344 \pm 0.056$ & 29 & 0.3 & 0.3 & 0.0 & 2.366 & 1.000 & $0.019^{+0.071}-0.019$ \\
\hline \multicolumn{11}{|c|}{ Check stars (all baselines) } \\
\hline HD 113226 & $3.066 \pm 0.008$ & $3.088 \pm 0.030$ & $3.018 \pm 0.054$ & $10 \pm 9$ & 1.8 & 1.8 & 0.0 & 3.053 & 0.996 & $0.023^{+0.028}-0.023$ \\
\hline HD 216131 & $2.369 \pm 0.010$ & $2.395 \pm 0.047$ & $2.333 \pm 0.047$ & 27 & 0.3 & 0.5 & 0.1 & 2.364 & 0.998 & $0.027^{+0.036}-0.027$ \\
\hline
\end{tabular}

Note. ${ }^{\text {a }}$ No error given for $\{a, b, \phi\}$ or oblateness $o_{a b}=a / b-1$ indicates that the error term was not constrained by examination of the $\Delta \chi_{\text {ellipse }}^{2}$ surface.

$\Delta \chi_{v}^{2}>1$ ) for only five of the objects: HIP 92194, HIP 95024, HIP 95777, HIP 102764, and HIP 105539. For our ensuing analyses, it is useful to note three statistics that we can establish with these five stars. First, we can compute the equivalent angular size by examining the area associated with an ellipse of dimensions $\{a, b\}$, where $\theta_{\text {equiv }}=\sqrt{a b}$. This parameter will allow us to proceed with the effective temperature analysis in Section 3.

Second, we can examine the ratio between the angular size indicated by $\theta_{\text {circ }}$ and the ellipse axes $\{a, b\}: a / \theta_{\text {circ }}=$ $0.954 \pm 0.041$ and $b / \theta_{\text {circ }}=1.046 \pm 0.021-$ overall, an average difference of $\sim 4.6 \%$. Thus, for those stars presented that have insufficient $\{u, v\}$ coverage (the seven other stars in Table 2, plus all other objects found in Table 1), we can establish an angular size error floor for circular UD fits of $\pm 4.6 \%$ : the formal $\theta_{\text {UD }}$ fitting error to the visibility data seen in Table 1 might be well below this value, but without better $\{u, v\}$ coverage, it is unclear where on the stellar photosphere the angular size data are being sampled.

Finally, the average oblateness for these five stars is $\overline{o_{a b}}=$ $\overline{a / b-1}=0.127 \pm 0.031$, which we will examine in Section 4.5 in considering the origins of non-spherical symmetry for these objects.

\subsubsection{Check Stars: Spherical Giants}

The indication that many carbon stars we have observed are non-spherical in a statistically significant manner (and that more may be, beyond the statistical significance of our measurements) is a remarkable finding. One necessary check of consistency is to examine similar data for stars that are, a priori, not expected to show any departures from spherical symmetry. To that end, we examined the PTI archive for giant stars that had sufficiently complete data sets for a similar analysis as that presented in Section 2.6.2. These objects were not necessarily observed on the same nights as the carbon stars in this study, but the data were collected in an identical queue-scheduled manner and reduced following an identical data reduction prescription. Additionally, we cross-referenced this list against the catalog of de Medeiros \& Mayor (1999) to select stars with small $v \sin i$ values.

For the purposes of demonstrating the integrity of our approach, we selected HD 113226 and HD 216131, which are G8III, G8+III giants (Gray et al. 2003; Keenan \& McNeil 1989) with rotational velocities of $v \sin i=1.2$ and $2.3 \mathrm{~km} \mathrm{~s}^{-1}$, respectively. These stars were selected purely on the criteria that (1) a proxy estimate (van Belle 1999) of their angular size indicated $3.0<\theta_{\mathrm{EST}}<4.0$ mas and (2) of all the giants in the PTI archive, these two objects had the greatest number of visibility points available in all three PTI configurations: NS, NW, and southwest (SW).

Using the same analysis as described for the carbon stars in Section 2.6.2, for just the NS and NW interferometry data, we fit the angular sizes of these two giants using both circular and elliptical fits (bottom of Table 2 and Figure 5). HD 113226 exhibits one clear outlier in the angular size data that is discarded. The expectation here is that if there was a systematic (and non-astrophysical) offset between the calibrated visibility points of the NS versus the NW baselines (which would manifest itself as artificial ellipticity), we would see elliptical fits for these two objects appearing (improperly) as statistically significant. As seen in Table 2, this fact is not the case: HD 113226 is robustly $\theta_{\text {circ }}=3.062 \pm 0.030$ and HD 216131 is $\theta_{\text {circ }}=2.366 \pm 0.031$, with no statistically significant to an elliptical fit in either case.

Additionally, these objects have the benefit of further $\{u, v\}$ coverage in the form of SW baseline data (Figure 6), which permit us one further level of consistency check. For this case, HD 216131 also exhibits one clear outlier in the angular size data that is discarded. The additional constraint of increased $\{u, v\}$ coverage improves the fit to the major axis $a$ in the elliptical fit for both objects, but in both cases, the elliptical fit continues to 

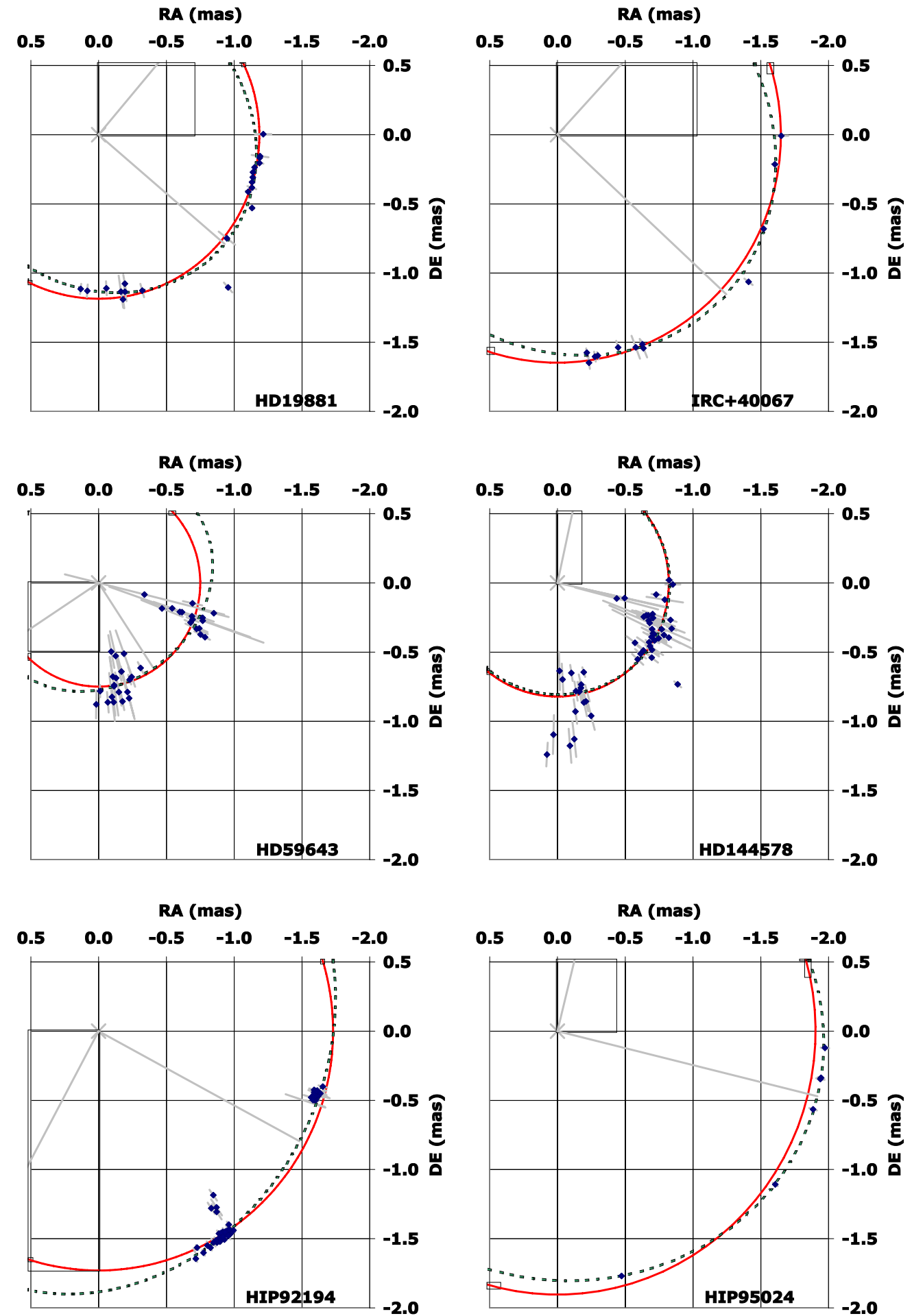

Figure 3. Stellar limb fits for the carbon stars in this study, including circular fits (red solid lines) and elliptical fits (green dotted lines). Outlier data points at $\{-0.9,-1.1\}$ mas for HD 19881 and $\{-0.9,-0.7\}$ mas for HD 144578 are not included in the fit data of Table 2; outliers at $\{-0.8,-1.3\}$ mas for HIP 92194 are also discarded.

(A color version of this figure is available in the online journal.)

show as no improvement over a simple circular fit; the fit for a simple circle is statistically unchanged with HD 113226 at $\theta_{\text {circ }}=3.066 \pm 0.034$ and HD 216131 is $\theta_{\text {circ }}=2.369 \pm 0.036$. Even though the SW baseline data were not taken for the carbon stars in this study, a comparison of the NS-NW giant stars fits relative to the NS-NW-SW fits illustrate the robust quality of the NS-NW fits, even with the lack of the additional $\{u, v\}$ constraint provided by the SW baseline data.

\section{EFFECTIVE TEMPERATURES}

\subsection{Bolometric Flux Estimates}

For each of the target stars observed in this investigation, a bolometric flux $\left(F_{\mathrm{BOL}}\right)$ estimate was established from the SED fits. This fit was accomplished using literature photometry values, with spectra from our carbon star models (Section 2.2; see example spectra in Figure 7). The model spectra were 

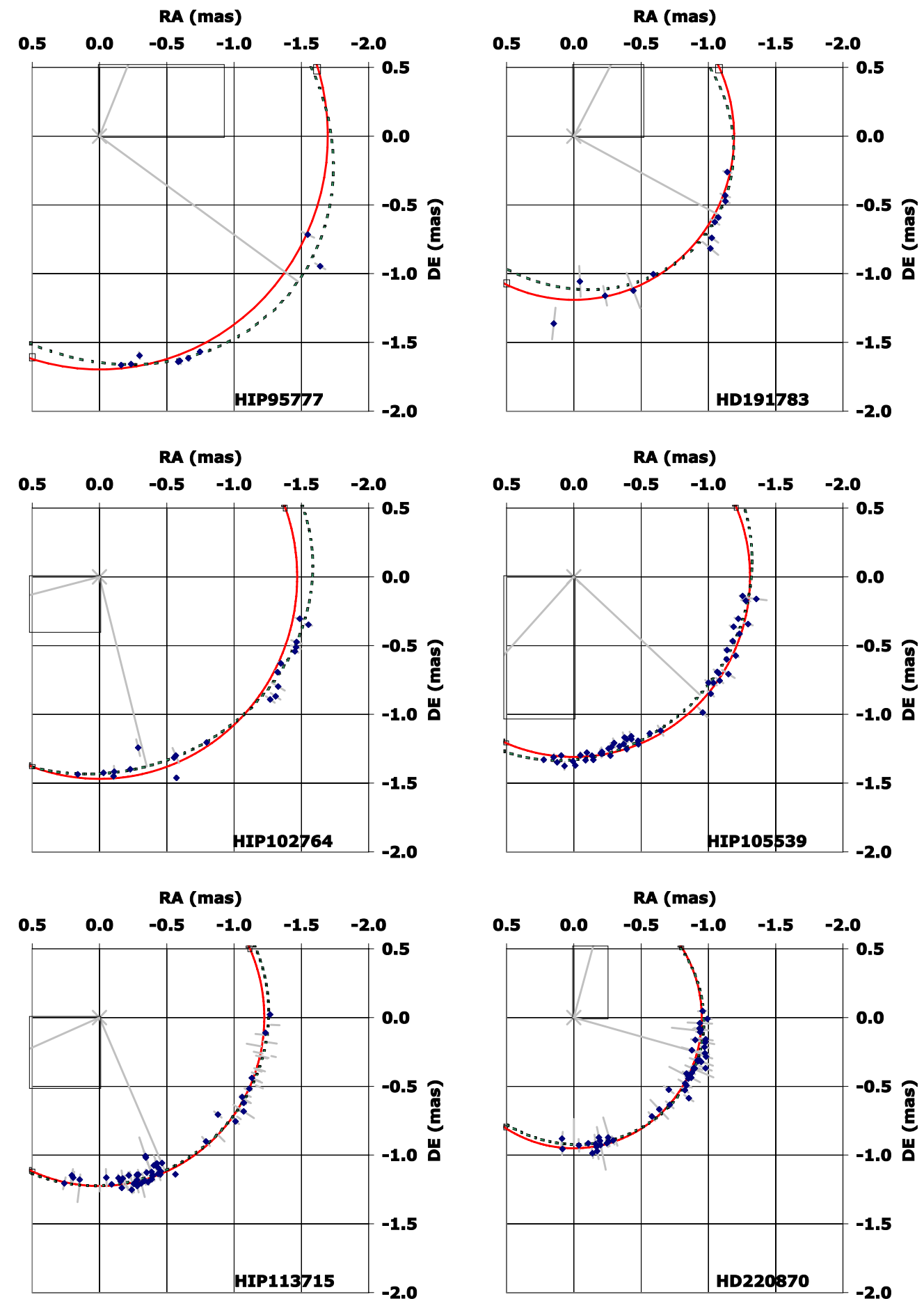

Figure 4. Stellar limb fits for the carbon stars in this study (continued), including circular fits (red solid lines) and elliptical fits (green dotted lines).

(A color version of this figure is available in the online journal.)

adjusted to account for overall flux level and wavelengthdependent reddening. Reddening corrections were based upon the empirical reddening determination described by Cardelli et al. (1989), which differs little from van de Hulst's theoretical reddening curve number 15 (Johnson 1968; Dyck et al. 1996a). Both narrowband and wide-band photometry over $0.5-25 \mu \mathrm{m}$ were used as available, including Johnson $U B V$ (see, for example, Eggen 1963, 1972; Moreno 1971), Stromgren $u b v y \beta$ (Piirola 1976), Geneva (Rufener 1976), Two Micron All Sky Survey $J_{H K}$ (Cutri et al. 2003), Vilnius UPXYZS (Zdanavicius et al. 1972), $V$ - and $I$ - band data from the TASS Mark IV survey (Droege et al. 2007), and the COBE DIRBE Point Source catalog (Smith et al. 2004), which included additional near-IR to mid-IR data from the Midcourse Space Experiment (MSX) satellite as well. Zero-magnitude flux density calibrations were based upon the values given in Fukugita et al. (1995) and Cox (2000), or the system reference papers cited above. The full list of photometry used for the SED fits is given in Table 3.

One concern regarding the photometry used herein is that it was not taken contemporaneously with the interferometric 

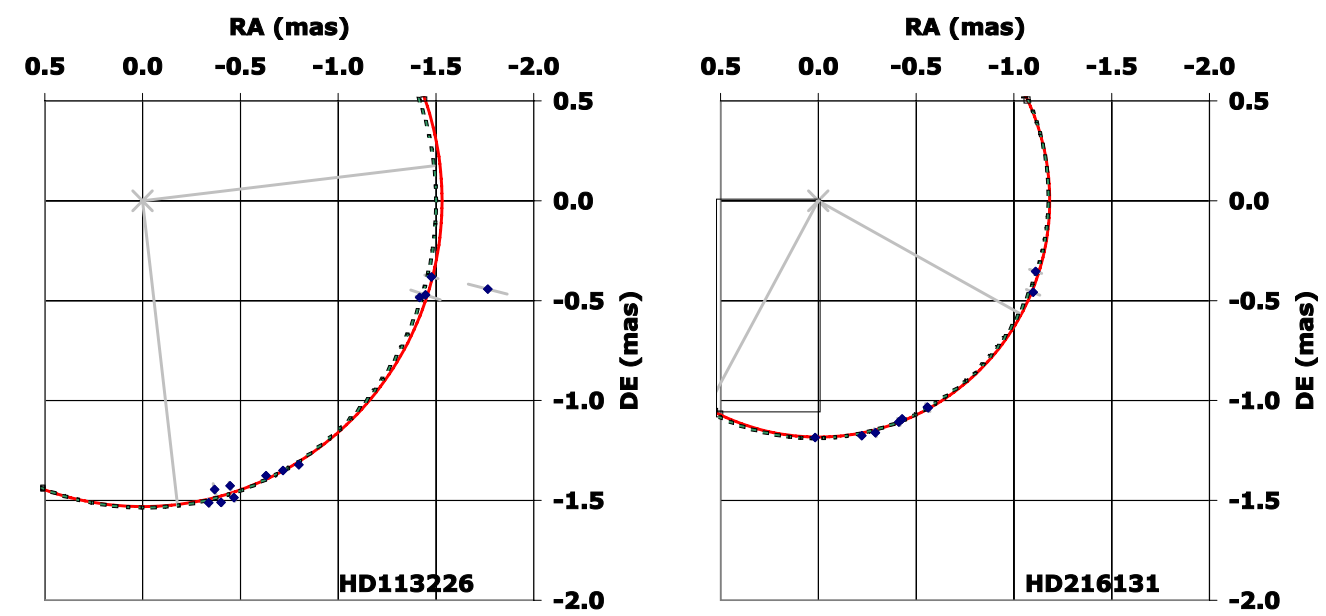

Figure 5. Angular size data for the slow-rotating giant check stars HD 113226 (G8III) and HD 216131 (G8+III) from the PTI NS and NW baselines; these stars have $v \sin i=1.2$ and $2.3 \mathrm{~km} \mathrm{~s}^{-1}$, respectively (de Medeiros \& Mayor 1999) and, as such, are expected to present circularly symmetric geometry upon the sky. This expectation is substantiated by the fit data in Table 2 .

(A color version of this figure is available in the online journal.)
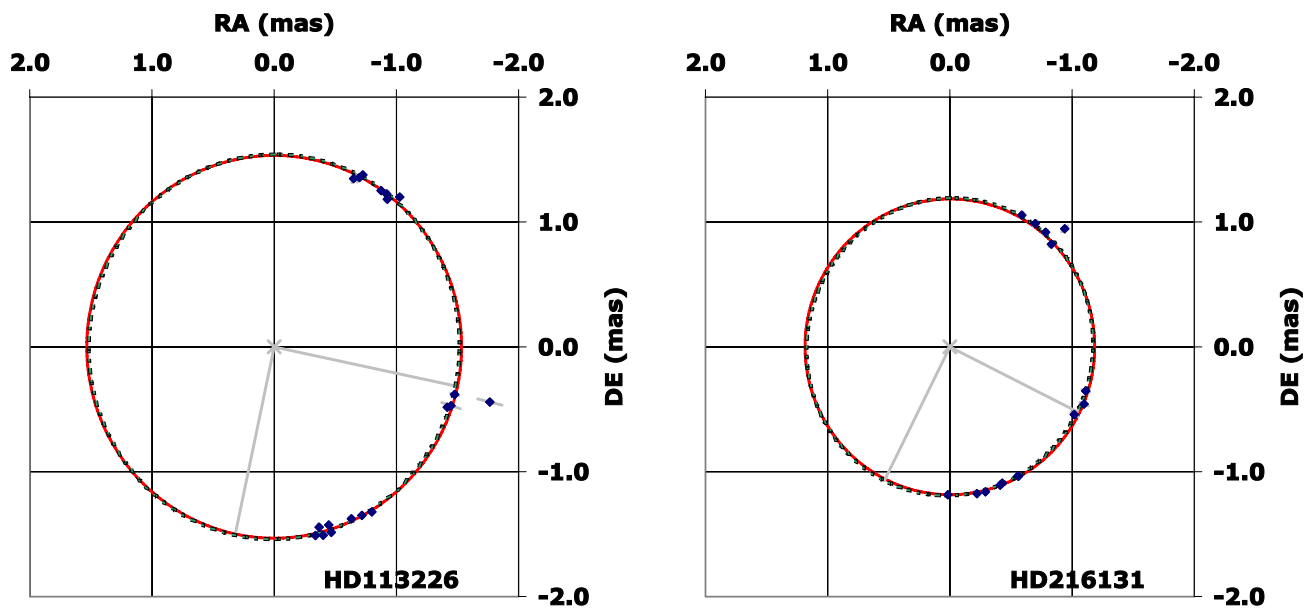

Figure 6. As in Figure 5 but including the additional SW baseline of the PTI. The outlier at $\{-1.0,+1.0\}$ mas for HD 216131 was discarded.

(A color version of this figure is available in the online journal.)

angular sizes. However, this concern is mitigated by noting a few properties of these particular objects. First, these are nonMira carbon stars and, as such, have lower levels of photometric variability in the visible than those extreme variables; the visible levels of variability are characterized for our target stars in Table 1.

Second, and more importantly, in the near-IR to mid-IR, where the bulk of the bolometric flux is emitted, these objects show significantly less variability. This result can be seen informally for those stars in Table 3 with multiple measurements over time-e.g., HD 33016 has $m_{K}=2.29,2.26$, and 2.31, HD 173291 has $m_{K}=1.69,1.64$, and 1.62-where the epoch-to-epoch change in the near-IR magnitudes is less than the measurement error (typically $\Delta m_{K}=0.1$ ). The study of Whitelock et al. (2006) found that for non-Mira carbon stars, the $K$-band variability was, on average, $\Delta \overline{m_{K}}=0.26$. (The Mira carbons in the same study showed significant $K$-band variability, with $\Delta \overline{m_{K}}=1.14$ ).

Since more than $50 \%$ of a carbon star's flux is emitted between the $J$ and $K$ bands (and over $90 \%$ of the flux is emitted between the $R$ and $L$ bands; see Figure 7), we are honing in on a significant fraction of the emitted flux of these objects. For those cases were $V$-band photometry was available from the General Catalog of
Variable Stars (GCVS; Samus et al. 2009), the V-band points reflect the average value between the minimum and maximum contained in the GCVS, with an uncertainty reflecting the range of $V$-band values. Overall, these uncertainties due to variability should not affect the general trends derived for carbon stars in Section 3.2.

As a sanity check on whether static model carbon star atmospheres are necessary for this evaluation, versus hydrostatic atmospheres, we can check the average effective temperatures derived in the next section for those stars in the lower half of visible light variability versus those in the upper half. Examining Table 1, we find our largest homogenous data set of visible light variability data is from the AFOEV (L'Association Française des Observateurs d'Étoiles Variables), ${ }^{8}$ with the separation occurring at $\Delta V=1.55 \mathrm{mag}$. For the less variable stars, we have 17 objects $^{9}$ with $\overline{T_{\mathrm{EFF}}}=2950 \pm 270 \mathrm{~K}$; for the more variable stars, we have 12 objects with $\overline{T_{\mathrm{EFF}}}=2850 \pm 280 \mathrm{~K}$-an indication that there is no significant difference based upon this metric.

\footnotetext{
8 http://cdsarc.u-strasbg.fr/afoev/

9 Omitting HD 59643, for the reasons discussed in Sections 4.1 and 4.2.
} 
HD2342 Net SED Model

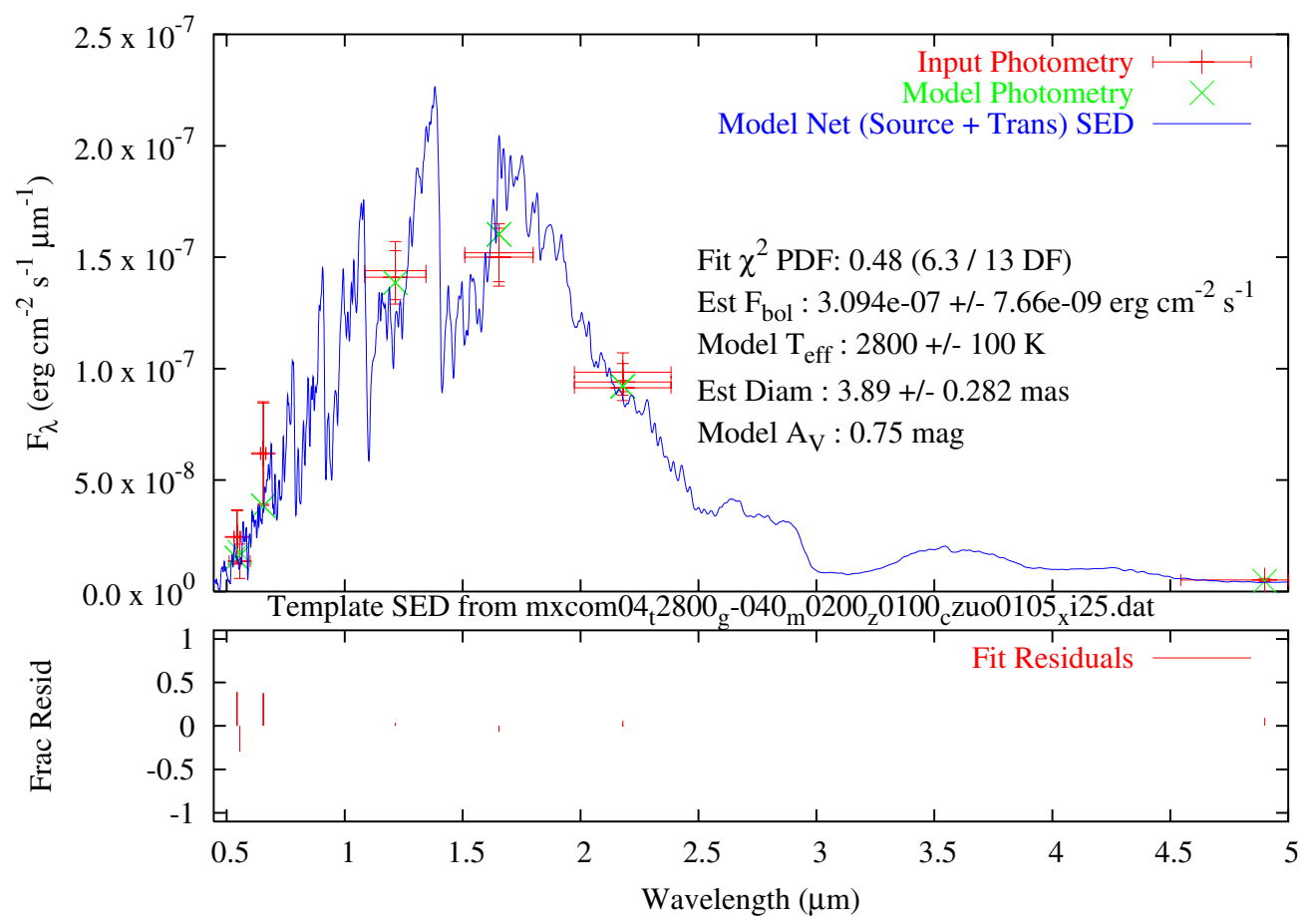

Figure 7. Representative spectra for a limb-darkened model atmosphere with $T_{\mathrm{EFF}}=2800 \mathrm{~K}, \log g=-0.40$, and $M=2 M_{\odot}$, fit to the HD 2342 photometry with $A_{V}=0.75$. The model atmosphere from Aringer et al. (2009).

(A color version of this figure is available in the online journal.)

Table 3

Target Photometry Used in SED Fits

\begin{tabular}{|c|c|c|c|c|}
\hline $\begin{array}{l}\text { Star } \\
\text { ID }\end{array}$ & $\begin{array}{c}\text { System/ } \\
\text { Wavelength }\end{array}$ & $\begin{array}{l}\text { Bandpass/ } \\
\text { Bandwidth }^{\mathrm{a}}\end{array}$ & $\begin{array}{c}\text { Value }^{\mathrm{b}} \\
(\mathrm{mag} / \mathrm{Jy})\end{array}$ & Reference \\
\hline HD 225217 & Johnson & V & $8.25 \pm 0.25$ & Samus et al. (2009) \\
\hline HD 225217 & Johnson & $J$ & $3.71 \pm 0.10$ & Noguchi et al. (1981) \\
\hline HD 225217 & Johnson & $H$ & $2.78 \pm 0.10$ & Noguchi et al. (1981) \\
\hline HD 225217 & Johnson & $K$ & $2.33 \pm 0.08$ & Neugebauer \& Leighton (1969) \\
\hline HD 225217 & Johnson & $K$ & $2.40 \pm 0.10$ & Bergeat et al. (1976) \\
\hline HD 225217 & Johnson & $K$ & $2.49 \pm 0.10$ & Bergeat \& Lunel (1980) \\
\hline HD 225217 & Johnson & $K$ & $2.34 \pm 0.10$ & Noguchi et al. (1981) \\
\hline HD 225217 & 4900 & 712 & $18.70 \pm 6.40$ & Smith et al. (2004) \\
\hline HD 225217 & 12000 & 6384 & $14.30 \pm 35.00$ & Smith et al. (2004) \\
\hline HD 2342 & Vilnius & V & $7.96 \pm 0.75$ & Straizys et al. (1989) \\
\hline HD 2342 & Vilnius & $V$ & $7.98 \pm 0.75$ & Straizys et al. (1989) \\
\hline HD 2342 & Johnson & $V$ & $10.85 \pm 0.95$ & Samus et al. (2009) \\
\hline HD 2342 & Vilnius & $S$ & $6.16 \pm 0.50$ & Straizys et al. (1989) \\
\hline HD 2342 & Vilnius & $S$ & $6.17 \pm 0.50$ & Straizys et al. (1989) \\
\hline HD 2342 & Johnson & $J$ & $3.43 \pm 0.10$ & Bergeat et al. (1976) \\
\hline HD 2342 & Johnson & $J$ & $3.40 \pm 0.10$ & Bergeat \& Lunel (1980) \\
\hline HD 2342 & Johnson & $H$ & $2.20 \pm 0.10$ & Bergeat et al. (1976) \\
\hline HD 2342 & Johnson & $H$ & $2.21 \pm 0.10$ & Bergeat \& Lunel (1980) \\
\hline HD 2342 & Johnson & $K$ & $1.64 \pm 0.04$ & Neugebauer \& Leighton (1969) \\
\hline HD 2342 & Johnson & $K$ & $1.56 \pm 0.10$ & Bergeat et al. (1976) \\
\hline HD 2342 & Johnson & $K$ & $1.61 \pm 0.10$ & Bergeat \& Lunel (1980) \\
\hline HD 2342 & 4900 & 712 & $41.40 \pm 5.60$ & Smith et al. (2004) \\
\hline HD 2342 & 12000 & 6384 & $20.50 \pm 19.20$ & Smith et al. (2004) \\
\hline
\end{tabular}

Notes. The collection of photometry used in the SED fitting routine for all objects. Refer to Section 3.1 for details.

${ }^{a}$ Wavelength is in $\mathrm{nm}$.

${ }^{\mathrm{b}}$ Values for system-bandpass data are in magnitudes; values for wavelength-bandwidth data are in Janskys.

(This table is available in its entirety in a machine-readable form in the online journal. A portion is shown here for guidance regarding its form and content.) 
Table 4

SED Fit Results and Derived Quantities

\begin{tabular}{|c|c|c|c|c|c|c|c|c|c|c|c|c|c|c|}
\hline \multirow{2}{*}{$\begin{array}{l}\text { Star } \\
\text { ID }\end{array}$} & \multirow{2}{*}{$\begin{array}{c}\text { Yamashita } \\
\text { Spectral } \\
\text { Type }^{\mathrm{a}}\end{array}$} & \multicolumn{3}{|c|}{ Model Parameters } & \multicolumn{3}{|c|}{ Fit Parameters } & \multicolumn{4}{|c|}{ Derived } & \multicolumn{3}{|c|}{ Previous } \\
\hline & & $\begin{array}{c}{\left[T_{\mathrm{EFF}} / \log g\right]} \\
\left(\mathrm{K} \mathrm{dex}^{-1}\right)\end{array}$ & $\begin{array}{c}R \\
\left(R_{\odot}\right)\end{array}$ & $\begin{array}{l}M_{\mathrm{BOL}} \\
\text { (mag) }\end{array}$ & $\begin{array}{c}A_{V} \\
\text { (mag) }\end{array}$ & $f_{\mathrm{BOL}}{ }^{\mathrm{b}}$ & $\chi_{\mathrm{RED}}^{2}$ & $\begin{array}{l}T_{\mathrm{EFF}} \\
(\mathrm{K})\end{array}$ & $\begin{array}{c}\Delta T^{\mathrm{c}} \\
(\mathrm{K})\end{array}$ & $\begin{array}{l}d_{R} \\
(\mathrm{pc})\end{array}$ & $\begin{array}{l}d_{\mathrm{BOL}} \\
(\mathrm{pc})\end{array}$ & $\begin{array}{l}T_{\mathrm{EFF}} \\
(\mathrm{K})\end{array}$ & $\begin{array}{l}\Delta T \\
(\mathrm{~K})\end{array}$ & Reference \\
\hline HD 225217 & $\mathrm{C} 6,4$ & {$[3000 /-0.6]$} & 467 & -5.78 & 0.75 & $17.7 \pm 0.60$ & 2.1 & $2989 \pm 102$ & -11 & 1684 & 1698 & 3057 & -68 & OT96 \\
\hline HD 2342 & C5, 4 & {$[2800 /-0.4]$} & 371 & -4.98 & 0.75 & $30.9 \pm 0.77$ & 0.5 & $2808 \pm 95$ & 8 & 892 & 887 & $2835 \pm 191$ & -27 & ОТ96, Т07 \\
\hline HD 19881 & $\mathrm{C} 7,4$ & {$[3300 /-0.2]$} & 295 & -5.19 & 2.25 & $22.3 \pm 0.75$ & 1.4 & $3314 \pm 113$ & 14 & 1162 & 1151 & 3247 & 67 & ОT96 \\
\hline IRC+40067 & C6, 3 & {$[2600 /-0.4]$} & 371 & -4.66 & 0.75 & $16.5 \pm 0.71$ & 2.2 & $2606 \pm 89$ & 6 & 1051 & 1047 & 2955 & -349 & OT96 \\
\hline HD 30443 & $\mathrm{C} 4,3 \mathrm{CH} 4: \mathrm{CN} 2: \mathrm{Ba} 4$ & {$[4000 / 0.0]$} & 234 & -5.53 & 2.50 & $15.5 \pm 0.57$ & 0.1 & $3884 \pm 161$ & -116 & 1521 & 1615 & 3580 & 304 & Т91 \\
\hline HD 280188 & $\mathrm{C} 8,1 \mathrm{~J}$ & {$[3300 /-0.2]$} & 295 & -5.19 & 2.50 & $49.1 \pm 2.59$ & 2.2 & $3361 \pm 114$ & 61 & 806 & 776 & & & \\
\hline HD 33016 & $\mathrm{C} 5,4$ & {$[2700 /-0.4]$} & 371 & -4.82 & 0.50 & $16.0 \pm 0.50$ & 0.8 & $2727 \pm 93$ & 27 & 1171 & 1147 & $3021 \pm 112$ & -294 & ОТ96, Т07 \\
\hline HD 34467 & $\mathrm{C} 6,3$ & {$[3000 /-0.2]$} & 295 & -4.78 & 1.00 & $13.6 \pm 0.68$ & 0.2 & $3003 \pm 102$ & 3 & 1224 & 1223 & 3207 & -204 & ОT96 \\
\hline HIP 25004 & $\mathrm{C} 4:, 4$ & {$[2700 /-0.8]$} & 588 & -5.82 & 2.25 & $19.5 \pm 0.82$ & 0.7 & $2715 \pm 92$ & 15 & 1664 & 1646 & & & \\
\hline HD 38218 & $\mathrm{C} 5,4$ & {$[2700 /-0.4]$} & 371 & -4.82 & 0.50 & $27.2 \pm 0.89$ & 0.4 & $2710 \pm 92$ & 10 & 885 & 878 & $2961 \pm 55$ & -251 & ОТ96, Т07 \\
\hline HD 247224 & $\mathrm{C} 5,4$ & {$[3100 /-0.4]$} & 371 & -5.42 & 1.00 & $7.5 \pm 0.34$ & 1.1 & $3056 \pm 104$ & -44 & 2152 & 2213 & & & \\
\hline HD 38572 & $\mathrm{C} 7,2$ & {$[3500 /-0.2]$} & 295 & -5.45 & 2.25 & $34.8 \pm 1.08$ & 1.4 & $3483 \pm 118$ & -17 & 1028 & 1039 & $3099 \pm 140$ & 384 & ОТ96, Т07 \\
\hline HD 38521 & $\mathrm{C} 4,4$ & {$[2600 /-0.4]$} & 371 & -4.66 & 1.25 & $8.0 \pm 0.55$ & 2.1 & $2580 \pm 88$ & -20 & 1479 & 1504 & & & \\
\hline HIP 29896 & C5, 4e: & {$[2600 /-0.6]$} & 467 & -5.16 & 2.25 & $18.6 \pm 0.66$ & 0.6 & $2597 \pm 88$ & -3 & 1240 & 1245 & 2735 & -138 & OT96 \\
\hline HD 45087 & $\mathrm{C} 5,4$ & {$[2700 /-0.6]$} & 467 & -5.32 & 2.00 & $13.9 \pm 0.61$ & 1.1 & $2723 \pm 92$ & 23 & 1575 & 1548 & & & \\
\hline HIP 31349 & $\mathrm{C} 8,3 \mathrm{e}$ & {$[3200 /-0.6]$} & 467 & -6.06 & 2.50 & $52.6 \pm 1.12$ & 0.7 & $3208 \pm 109$ & 8 & 1123 & 1118 & & & \\
\hline HD 46321 & $\mathrm{C} 4,5$ & {$[2800 /-0.4]$} & 371 & -4.98 & 0.75 & $9.2 \pm 0.48$ & 0.2 & $2794 \pm 95$ & -6 & 1622 & 1630 & & & \\
\hline HD 47883 & $\mathrm{C} 5,4 \mathrm{Ba} 5$ & {$[3100 /-0.2]$} & 295 & -4.92 & 0.75 & $15.0 \pm 0.73$ & 0.9 & $3118 \pm 106$ & 18 & 1252 & 1238 & 3236 & -118 & OT96 \\
\hline HD 48664 & $\mathrm{C} 4,5$ & {$[2600 /-0.6]$} & 467 & -5.16 & 1.25 & $12.9 \pm 0.63$ & 0.6 & $2612 \pm 89$ & 12 & 1505 & 1494 & 2860 & -248 & OT96 \\
\hline HD 5 & $\mathrm{C} 4,4 \mathrm{MS} 2$ & {$[3300 /-0.2]$} & 295 & -5.19 & 0.75 & $45.7 \pm 2.09$ & 0.6 & $3290 \pm 112$ & -10 & 800 & 804 & 3330 & -40 & ОТ96 \\
\hline HD 54361 & C6, 3 & {$[2900 / 0.0]$} & 234 & -4.13 & 0.50 & $56.8 \pm 1.95$ & 1.0 & $2907 \pm 99$ & 7 & 445 & 443 & $3085 \pm 290$ & -178 & L86, OT96 \\
\hline IRC+10158 & $\mathrm{C} 5,5$ & {$[2500 /-0.6]$} & 467 & -4.99 & 1.25 & $12.4 \pm 0.61$ & 1.9 & $2561 \pm 87$ & 61 & 1473 & 1406 & & & \\
\hline HD 57160 & $\mathrm{C} 5,4 \mathrm{~J}$ & {$[2900 /-0.2]$} & 295 & -4.63 & 0.50 & $11.1 \pm 0.45$ & 3.1 & $2871 \pm 98$ & -29 & 1237 & 1262 & $3105 \pm 134$ & -234 & ОТ99, Т07 \\
\hline HD 59643 & C6, 2 CH3 & {$[3800 / 0.0]$} & 234 & -5.31 & 1.50 & $15.9 \pm 0.42$ & 1.5 & $3775 \pm 128$ & -25 & 1420 & 1442 & $3351 \pm 71$ & 425 & От96, Z09 \\
\hline HD 60826 & $\mathrm{C} 5,5$ & {$[2700 /-0.2]$} & 295 & -4.32 & 0.75 & $10.1 \pm 0.46$ & 3.5 & $2694 \pm 92$ & -6 & 1143 & 1147 & & & \\
\hline HD 70072 & $\mathrm{C} 6,4 \mathrm{e}$ & {$[2400 /-0.4]$} & 371 & -4.31 & 1.50 & $18.1 \pm 0.46$ & 1.9 & $2381 \pm 81$ & -19 & 838 & 852 & $2632 \pm 45$ & -251 & ОТ96, Т07 \\
\hline HD 144578 & $\mathrm{C} 8,1 \mathrm{e}$ & {$[3500 / 0.0]$} & 234 & -4.95 & 1.25 & $11.8 \pm 1.09$ & 4.0 & $3450 \pm 117$ & -50 & 1375 & 1416 & & & \\
\hline HD 173291 & $\mathrm{C} 7,4$ & {$[2800 /-0.4]$} & 371 & -4.98 & 0.75 & $31.5 \pm 1.01$ & 0.8 & $2793 \pm 95$ & -7 & 874 & 879 & 2866 & -73 & OT96 \\
\hline HIP 92194 & & {$[2800 /-0.6]$} & 467 & -5.48 & 2.25 & $24.4 \pm 0.96$ & 0.8 & $2803 \pm 95$ & 3 & 1259 & 1257 & & & \\
\hline HIP 95024 & $\mathrm{C} 4,5 \mathrm{e}$ & {$[2500 /-0.6]$} & 467 & -4.99 & 1.50 & $19.6 \pm 0$ & 3.6 & $2483 \pm 84$ & -17 & 1104 & 1121 & 2702 & -219 & OT96 \\
\hline HIP 95777 & $\mathrm{C} 4,5$ & {$[2800 /-0.6]$} & 467 & -5.48 & 1.00 & $23.4 \pm 0.77$ & 1.0 & $2798 \pm 95$ & -2 & 1282 & 1284 & 2759 & 39 & OT96 \\
\hline HD 186047 & C6.5, y & {$[2900 / 0.0]$} & 234 & -4.13 & 0.50 & $25.0 \pm 0.57$ & 1.9 & $2878 \pm 98$ & -22 & 658 & 668 & 3046 & -168 & OT96 \\
\hline HIP 99336 & $\mathrm{C} 6,3 \mathrm{e}$ & {$[3000 /-0.4]$} & 371 & -5.28 & 2.00 & $11.3 \pm 0.60$ & 1.3 & $2924 \pm 113$ & -76 & 1599 & 1685 & & & \\
\hline HD 191783 & $\mathrm{C} 6,4$ & {$[2800 /-0.4]$} & 371 & -4.98 & 0.75 & $11.5 \pm 0.45$ & 1.0 & $2796 \pm 95$ & -4 & 1447 & 1453 & & & \\
\hline HIP 102764 & C6, 4 & {$[2700 / 0.0]$} & 234 & -3.82 & 0.75 & $15.0 \pm 0.56$ & 1.3 & $2705 \pm 92$ & 5 & 750 & 746 & & & \\
\hline HIP 105539 & $\mathrm{C} 6,3 \mathrm{e}$ & {$[2700 /-0.4]$} & 371 & -4.82 & 1.00 & $11.8 \pm 0.82$ & 1.2 & $2703 \pm 92$ & 3 & 1338 & 1335 & & & \\
\hline IRC+50399 & $C 7, y$ & {$[3000 /-0.6]$} & 467 & -5.78 & 2.25 & $11.6 \pm 0.48$ & 1.5 & $3006 \pm 103$ & 6 & 2097 & 2090 & & & \\
\hline HD 208512 & C5, 4e: & {$[2700 /-0.8]$} & 588 & -5.82 & 1.25 & $27.3 \pm 1.04$ & 1.8 & $2691 \pm 91$ & -9 & 1382 & 1390 & 2500 & 191 & Z09 \\
\hline HD 208526 & C4, 4J MS5 & {$[2800 / 0.0]$} & 234 & -3.98 & 0.75 & $18.4 \pm 0.67$ & 1.4 & $2804 \pm 95$ & 4 & 728 & 726 & 2890 & -86 & OT99 \\
\hline HIP 113715 & $\mathrm{C} 3,5 \mathrm{~J}$ j6 MS6 & {$[2500 /-0.2]$} & 295 & -3.99 & 0.75 & $8.0 \pm 0.21$ & 0.9 & $2510 \pm 85$ & 10 & 1115 & 1108 & & & \\
\hline HD 220870 & $\mathrm{C} 7,3$ & {$[2900 /-0.2]$} & 295 & -4.63 & 0.50 & $8.4 \pm 0.35$ & 0.9 & $2886 \pm 98$ & -14 & 1438 & 1451 & & & \\
\hline
\end{tabular}

Notes. Additional non-variable model parameters were $M=2 M_{\odot}, Z / Z_{\odot}=1$, and $\mathrm{C} / \mathrm{O}=1.05$.

${ }^{a}$ Values in italics predicted from measured $T_{\mathrm{EFF}}$ and Equation (2) for those stars (HD 186047, IRC+50399) for which no Yamashita spectra type was given.

${ }^{\mathrm{b}}$ In units of $10^{-8} \mathrm{erg} \mathrm{cm}^{-2} \mathrm{~s}^{-1}$. See the discussion of $F_{\mathrm{BOL}}$ error in Section 3.1.

${ }^{c}$ Difference between model $T_{\mathrm{EFF}}^{M}\left(\right.$ Column 3 ) and measured $T_{\mathrm{EFF}}$ (Column 9), derived from $\theta$ (Table 1$)$ and $F_{\mathrm{BOL}}($ Column 7).

References. L86: Lambert et al. 1986; T91: Tsuji et al. 1991; OT96: Ohnaka \& Tsuji 1996; OT99: Ohnaka \& Tsuji 1999; T07: Tanaka et al. 2007, Z09: Zamora et al. 2009.

For each star under consideration, the collected photometry was fit to a full grid of the model spectra, with an additional parameter of reddening being tested in steps of $\Delta A_{V}=0.25$ from $A_{V}=0.5$ to 2.5 . By selecting the result with the best reduced $\chi_{v}^{2}$, this fitting procedure resulted in a selection of the optimal model in terms of the parameters $T_{\mathrm{EFF}}$ and $\log g$; the fitting itself also provided fit values for the reddening $A_{V}$ and the source bolometric flux $F_{\mathrm{BOL}}$.

Thus, in Table 4, a value for $F_{\mathrm{BOL}}$ is presented for each star based upon a best fit achieved through searching a grid of $\left\{T_{\mathrm{EFF}}, \log g, A_{V}\right\}$; the $\chi_{v}^{2}$ value of that fit is also presented in Table 4. The values of the linear radius $R$ and the bolo- metric magnitude $M_{\mathrm{BOL}}$ associated with the best-fit model are also noted in Table 4. These models follow the usual mass-radius-luminosity relationships, $L=4 \pi R^{2} \sigma T_{\mathrm{EFF}}^{4}=$ $4 \pi(G M / g) \sigma T_{\mathrm{EFF}}^{4}$; as such, for a constant $T_{\mathrm{EFF}}$ and $g, \Delta L$ will scale linearly with $\Delta M, \Delta R$ scales $\Delta M^{-1 / 2}$, and $\Delta M_{\mathrm{BOL}}$ scales as $2.5 \log (\Delta M)$. As noted in Section 2.2, models with a $\mathrm{C} / \mathrm{O}$ ratio of 1.05 were used; to simplify the search grid, available models with $\mathrm{C} / \mathrm{O}=1.10,1.40$, and 2.00 were not. However, we tested these models to ascertain the impact on the derived $F_{\mathrm{BOL}}$ values as $\mathrm{C} / \mathrm{O}$ was varied; across a range of $T_{\mathrm{EFF}}$ values, for $\mathrm{C} / \mathrm{O}=1.10$ and $1.40, \Delta F_{\mathrm{BOL}}$ was less than $2 \%$, well within the $\sim 4 \% F_{\mathrm{BOL}}$ errors reported in Table 4. Figure 7 illustrates this 
result well: although a changing $\mathrm{C} / \mathrm{O}$ ratio will change the fine details of the SED, the gross nature of the SED will remain the same and, as such, as will the computed $F_{\mathrm{BOL}}$ from this procedure: Figure 3 of Aringer et al. (2009) illustrates this fact further.

Using the distances we derive in Section 3.3, the average rate of reddening for this ensemble is $A_{V}=1.06 \mathrm{mag} \mathrm{kpc}^{-1}$. In comparison with the "canonical" value of $0.7-1.0 \mathrm{mag}$ $\mathrm{kpc}^{-1}$ (Gottlieb \& Upson 1969; Milne \& Aller 1980; Lynga 1982), this value is on the high end of the expected envelope, although it is worth noting that most of these targets are near the Galactic plane with an average absolute Galactic latitude of $|\bar{b}|=10^{\circ} \pm 9^{\circ}$, where greater levels of extinction are expected. Circumstellar reddening is a significant phenomenon for these stars, and is accounted for in the inclusion of midIR photometric data from IRAS and DIRBE/MSX (Smith et al. 2004) - but is not significant factor (less than $1 \%$ of the total) when computing $F_{\mathrm{BOL}}$ : again, the dominant bandpass when integrating under the SED curve for such a determination is 1-2 $\mu \mathrm{m}$ (see Figure 7). As an independent check, $A_{V}$ was estimated using the three-dimensional map of Gontcharov (2012) (noting these stars are $\sim 1.5 \times$ more distant than the $1 \mathrm{kpc}$ calibration stars of that study). On average, our calculations of $A_{V}$ are slightly redder, but the comparison is framed by a considerable amount of scatter, with $\Delta A_{V}=0.22 \pm 0.83$. Given that these objects emit the principal portion of their flux redward of the $V$ band, in the 1.0-2.0 $\mu \mathrm{m}$ regime (as illustrated in Figure 7) where reddening drops markedly (i.e., $A_{K} \simeq 0.11 A_{V}$ ), we find that this low value of $\Delta A_{V}$ indicates our $F_{\mathrm{BOL}}$ values should, on average, be accurate.

We note an important fact regarding the errors reported on $F_{\mathrm{BOL}}$ : the uncertainties reported with the $F_{\mathrm{BOL}}$ values are the formal values from the fitting routine for an individual spectrum; however, these values probably underrepresent the actual uncertainty, with an average uncertainty of $\sim 4 \%$. As such, a limit of $10 \%$ uncertainty was taken as the actual $F_{\mathrm{BOL}}$ error for computation of the derived quantity $T_{\mathrm{EFF}}$ in Section 3.2. Such a limit is consistent with the derived values of $F_{\mathrm{BOL}}$ even in the presence of modest deviations in the model parameters (e.g., using a $T_{\mathrm{EFF}}=2800 \mathrm{~K}$ model when a $T_{\mathrm{EFF}}=3000 \mathrm{~K}$ model is appropriate, $\Delta \log g= \pm 0.20, \Delta A_{V}= \pm 0.25$, etc.). Additionally, we expect that this more conservative approach to the $F_{\mathrm{BOL}}$ uncertainty will be more consistent with the non-contemporaneous nature of the photometric data and the variability documented in Whitelock et al. (2006) for non-Mira carbon stars.

\subsection{Effective Temperatures}

Stellar effective temperature is defined in terms of a star's luminosity $(L)$ and linear radius $(R)$ by $L=4 \pi \sigma R^{2} T_{\mathrm{EFF}}^{4}$, which can be rewritten in terms of the stellar angular diameter and source bolometric flux: $T_{\mathrm{EFF}} \propto\left(F_{\mathrm{BOL}} / \theta^{2}\right)^{1 / 4}$. Inherent in the expression for $F_{\mathrm{BOL}}$ are the effects of interstellar reddening, hence the attention paid to the subject in the derivation of $F_{\mathrm{BOL}}$ in Section 3.1. As noted in Section 2.6.2, an angular size error floor of $\pm 4.6 \%$ was used (regardless of the formal fitting error in Table 1) due to uncharacterized stellar oblateness.

The derived values of $T_{\mathrm{EFF}}$ are presented in Table 4, along with the difference $(\Delta T)$ between these values and the input spectral model $T_{\mathrm{EFF}}^{M}$. On average, the difference between those two temperatures is $0.25 \sigma$, indicating a consistency in the overall approach. For these stars, the average error in determination of $T_{\mathrm{EFF}}$ is $100 \mathrm{~K}$ - a factor of $2.4 \times$ improvement over the previous large interferometric study of Dyck et al. (1996b). Comparisons with previous studies that have measured $T_{\mathrm{EFF}}$ by other methods for carbon stars (Lambert et al. 1986; Tsuji et al. 1991; Ohnaka \& Tsuji 1996, 1999; Tanaka et al. 2007; Zamora et al. 2009) show 23 stars in common (the rightmost columns of Table 4), with an average agreement at the level of $\overline{\Delta T_{\mathrm{EFF}}}=-67 \pm 215 \mathrm{~K}$.

Overall, the median temperature of this group is $T_{\mathrm{EFF}}=$ $2803 \pm 337 \mathrm{~K}$. If we exclude the "hot carbon" (HC) stars HD 59643 and HD 30443 (see the discussions in Sections 4.1 and 4.2), the median remains largely the same but the spread drops considerably with $T_{\mathrm{EFF}}=2798 \pm 268 \mathrm{~K}$.

We can also compare the four stars (HIP 31349/CR Gem, HD 51620/RV Mon, HD 173291/HK Lyr, and HIP 92194/ DR Ser) in common between this study and our previous study (Paladini et al. 2011), which used a spectral analysis approach for the determination of $T_{\mathrm{EFF}}$ (e.g., see Section 4.1 of that paper). Overall, the agreement is at the $1 \sigma$ level, with the average $T_{\mathrm{EFF}}$ for those four stars being $3082 \pm 74 \mathrm{~K}$ from that paper and $2989 \pm 51 \mathrm{~K}$ herein.

\subsection{Distances and Linear Radii}

A distance determination was possible for each star in our sample via two separate methods. First, through comparison of the measured angular size with the linear size associated with the best-fit model, a distance $\left(d_{R}\right)$ can be simply and directly extracted. Second, each model has an absolute bolometric magnitude $\left(M_{\mathrm{BOL}}\right)$ associated with it as well; by comparison of $M_{\mathrm{BOL}}$ with the unreddened apparent bolometric flux, a bolometric distance $\left(d_{\mathrm{BOL}}\right)$ can also be estimated. The values associated with these derivations can be found in Table 4.

These two separate (although not entirely independent) methods produce distances that agree well with each other-on average, only a $1 \% \pm 7 \%$ difference between $d_{R}$ and $d_{\mathrm{BOL}}$ is found. A more independent check is a comparison with the distances found in Claussen et al. (1987): for the 35 objects in common, the agreement is $2 \% \pm 20 \%$ - still very good on average, although with more scatter for individual objects. This scatter is perhaps connected to the somewhat general approach of Claussen et al. (1987) for distance determination; the assumption of that investigation was that all carbon stars had the same $2.2 \mu \mathrm{m}$ luminosity, with $M(K)=-8.1$, an average derived from 54 Large Magellanic Cloud (LMC)/Small Magellanic Cloud (SMC) carbon stars in Frogel et al. (1980) and Cohen et al. (1981) and assumed distance moduli of $18.6 \mathrm{mag}$ and $19.1 \mathrm{mag}$ for the LMC and SMC, respectively.

Although entries exist for 32 of these objects in the Hipparcos catalog, we chose not to use these data: the average fractional error $\left(|\pi| / \sigma_{\pi}\right)$ was $173 \%$. The average parallax error was $\sigma_{\pi}=1.3$ mas for these stars with $H_{p}=8.8 \pm 1.0$ and $B-V=$ $2.5 \pm 0.5$ (parameters typical of carbon stars), markedly greater than the average of $\sigma_{\pi}=0.65$ mas for stars of similar brightness, but with $B-V=0.5 \pm 0.5$ (typically A- to K-type mainsequence stars). As noted in Section 3.5 of van Belle et al. (2002) for the similar case of Mira variables, comparison of the diameter data of Table 1 and the distance information in Table 4 indicates that, on average, the angular sizes of these objects are $3.4 \times$ larger than their parallaxes, making such measurements particularly difficult (and, in Hipparcos's visible bandpass, susceptible to surface morphology, which should evolve on timescales comparable to the parallax measures.)

The median linear radii of these carbon stars is $R=$ $360 \pm 100 R_{\odot}$, although it should be emphasized that the values 


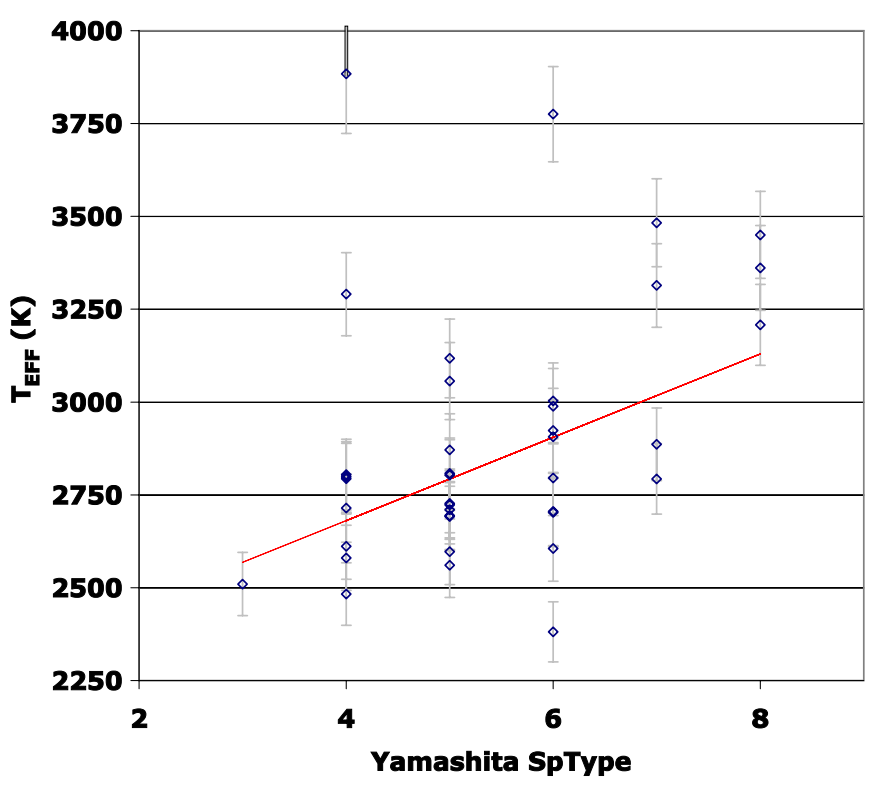

Figure 8. Comparison of temperature vs. spectra types for the common sample of 40 stars, from Yamashita $(1972,1975 b)$. The fit line excludes the two CH star outliers HD 30443 and HD 59643 above $3750 \mathrm{~K}$ and is described in Equation (2).

(A color version of this figure is available in the online journal.)

of this parameter are based upon the models and not the angular size data (since distances to carbon stars remain quite uncertain).

\section{DISCUSSION}

This large, homogenous data set allows us a number of interesting avenues to explore the implications for carbon stars. First, we can compare to previous studies (Yamashita 1972, 1975b; Bergeat et al. 2001, 2002a, 2002b). Second, we can use the new data to calibrate our expectations for angular size prediction for these stars. The indication that some carbon stars are oblate is then examined in some detail, including connections to the stellar angular momentum history and mass loss. Finally, the proposition that most carbon stars could be oblate is considered.

\subsection{Comparison with Yamashita (1972, 1975b) Spectral Types}

Yamashita $(1972,1975 b)$ spectra types are written as $C x, y$, where the criteria for sub-types $x$ and $y$ are described in detail in those investigations. The $x$ index quantifies the D-lines of $\mathrm{Na}$ I at $5890 \AA$ and $5896 \AA ; y$ is indicative of the strength of the Swan bands of $C_{2}$ at $5635 \AA$ and $6191 \AA$. The classification scheme of Yamashita is largely based upon (and intended to retain the principal features of) Keenan \& Morgan (1941); the $x$ index was intended to establish a temperature sequence, where $T_{\mathrm{EFF}}$ was expected to decrease with increasing $x$. However, an anti-correlation of $T_{\mathrm{EFF}}$ with the $x$ parameter is expected and has tentatively been detected in previous studies (Tsuji 1981; Dyck et al. 1996b). For our target stars, these carbon spectral types can be found in Table 4 and a plot of the $T_{\mathrm{EFF}}$ versus subtype $x$ can be seen in Figure 8 .

A straight fit to our spectral type data gives the relationship between subtype $x$ and $T_{\mathrm{EFF}}$ :

$$
T_{\mathrm{EFF}}=(112 \pm 13.2) x+(2231 \pm 71.6)
$$

with $\chi_{v}^{2}=7.71$ and an average temperature offset of $\overline{|\Delta T|}=$ $203 \mathrm{~K}$. If we exclude the obvious outliers HD 30443 and
Table 5

Summary of $T_{\mathrm{EFF}}$ Expectations for Yamashita Spectral Types

\begin{tabular}{lccc}
\hline \hline $\begin{array}{l}\text { Yamashita } \\
\text { Spectral Type } x\end{array}$ & $N$ & $\begin{array}{c}T_{\mathrm{EFF}, \mathrm{wtd}}{ }^{\mathrm{a}} \\
(\mathrm{K})\end{array}$ & $\begin{array}{c}T_{\mathrm{EFF}, \mathrm{fit}} \\
(\mathrm{K})\end{array}$ \\
\hline 3 & 1 & 2510 & 2534 \\
4 & 8 & $2726 \pm 247$ & 2650 \\
5 & 12 & $2763 \pm 168$ & 2767 \\
6 & 9 & $2746 \pm 207$ & 2883 \\
7 & 4 & $3067 \pm 337$ & 3000 \\
8 & 3 & $3334 \pm 123$ & 3116 \\
\hline
\end{tabular}

Note. ${ }^{\text {a }}$ Weighted average of $T_{\mathrm{EFF}}$ of those stars with a corresponding $x$-index in the $C x, y$ Yamashita spectral type.

HD 59643 above $T_{\mathrm{EFF}}>3750 \mathrm{~K}$-justified by the presence of the $\mathrm{CH}$ marker in their Yamashita spectral types-there is little change in the parameters of the fit:

$$
T_{\mathrm{EFF}}=(117 \pm 13.3) x+(2183 \pm 72.2)
$$

although the statistics of the fit improve markedly, with $\chi_{v}^{2}=$ 5.16 and $\overline{|\Delta T|}=152 \mathrm{~K}$. CH stars are thought to be a distinct group of high-velocity carbon stars (Yamashita 1975a, and references therein), which justifies this exclusion.

As evidenced by the poor $\chi_{v}^{2}$ value of the fit in Equation (2) and illustrated in Figure 8, the usefulness of $T_{\mathrm{EFF}}$ derived from the spectral index is less than optimal. A summary is presented in Table 5 including the $T_{\mathrm{EFF}}$-weighted average by spectral type and the $T_{\mathrm{EFF}}$ line fit value from Equation (2); a comparison of these summary values with the previous values in Table 4 of Dyck et al. (1996b) shows favorable agreement between these two studies. ${ }^{10}$

\subsection{Comparison with Bergeat et al. (2001, 2002b)}

We can compare our $T_{\mathrm{EFF}}$ determinations with the SED determinations found in Bergeat et al. (2002a), whose sample overlaps with 38 of our stars. As seen in Figure 9, the agreement is quite good up to $3000 \mathrm{~K}$, with the average difference being $0.21 \sigma$-our ensemble indicating temperatures on average only $17 \mathrm{~K}$ lower, well within the margin of error; the average absolute temperature difference in this range is $131 \mathrm{~K}$. However, for our temperatures above $3000 \mathrm{~K}$, our stars indicate temperatures on average $92 \mathrm{~K}$ hotter, possibly indicating a preference in the Bergeat et al. (2002a) process for $T_{\mathrm{EFF}}$ results below $3000 \mathrm{~K}$. It is difficult to isolate the source of this discrepancy, however; tracing the calibration of that study to Bergeat et al. (2001), we find their SED calibrations are grounded in angular size determinations. Indeed, it appears that 17 of the 54 diameter measurements in Bergeat et al. (2001) were preliminary PTI diameters from a conference proceeding of ours (van Belle \& Thompson 1999). While the quality of the measurements has

\footnotetext{
10 As suggested by our referee, it would be an interesting task to investigate the relationship of the spectral index $x$ versus $T_{\mathrm{EFF}}$ based on codes such as MOOG (Sneden 1973) or SYNTHE (Kurucz \& Avrett 1981). However, the sodium lines used to determine the $x$ index are very strong - thus, they are partly formed in the outer regions of the stellar atmosphere. This fact makes them very sensitive to deviations from hydrostatic equilibrium-especially to the appearance of even weak stellar winds - and to non-local thermal equilibrium (non-LTE) effects. Consequently, it is quite problematic to model these lines based on the hydrostatic LTE atmospheres utilized in this investigation or the MOOG/SYNTHE codes that also have underlying LTE assumptions. Additionally, we caution the interested investigator that the behavior of these lines will not relate to the parameters of the stars in a simple way, since what will be measured is a combination of effective temperature, shock intensities, mass-loss rates, atmospheric extension, etc.
} 


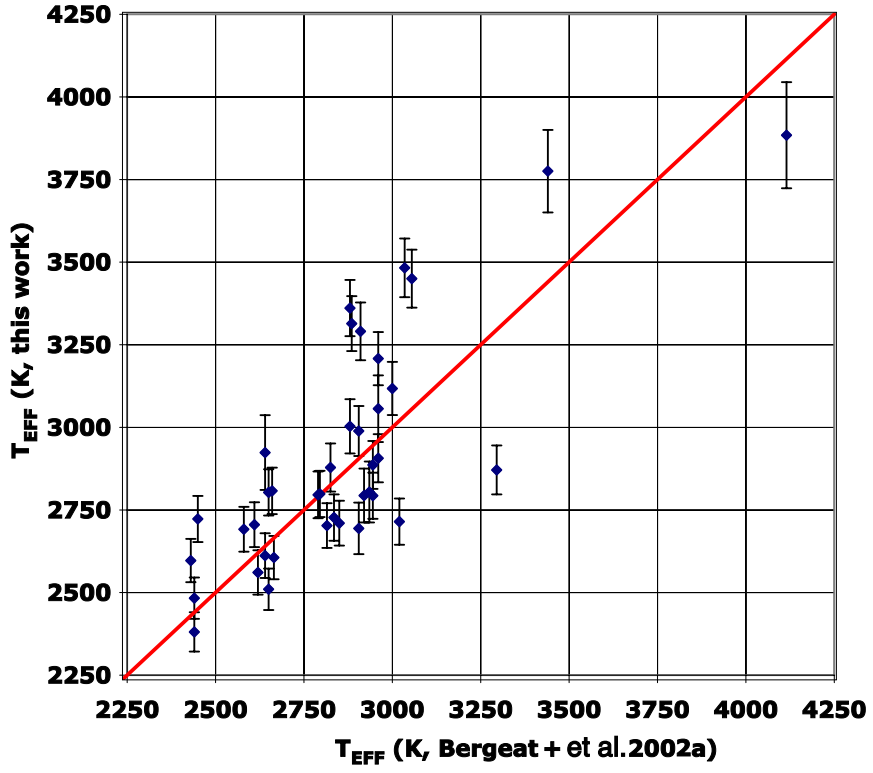

Figure 9. Comparison of effective temperatures for the sample of 38 stars in common with the Bergeat et al. (2002a) sample; the red line is the 1:1 relationship.

(A color version of this figure is available in the online journal.)

improved - the angular size errors are $\sim 3 \times$ smaller-the indicated angular sizes have, on average, not changed significantly. Overall, though, the average difference is only $15 \mathrm{~K}$, with an average absolute temperature difference of $153 \mathrm{~K}$.

Bergeat et al. (2001) utilize the photometric classification scheme proposed in Knapik \& Bergeat (1997) to classify carbon stars into six groups, CV1-CV6, and extended this classification to "hot" carbon stars groups HC1-HC5 in Bergeat et al. (1999). These groups are each represented by characteristic unreddened $B-V, C I_{\mathrm{B}}=[0.78]-[1.08]$, and $J-K$ colors (see Table 1 of Knapik \& Bergeat 1997).

As seen in Table 7 of Bergeat et al. (2001), $T_{\mathrm{EFF}}$ determinations for these groups, from angular sizes, are listed alongside similar such determinations from SEDs and calibrated color indices. The latter two $T_{\mathrm{EFF}}$ determinations are systematically lower in temperature for a given group than the former, particularly for temperatures above $3000 \mathrm{~K}$; the final adopted $T_{\mathrm{EFF}}$ scale reflects this lower scale as well.

On the highest temperature end $\left(T_{\mathrm{EFF}}>3400 \mathrm{~K}\right)$, it is significant to note that the two objects visible in Figure 9, HD 30443 and HD 59643, are HC4 and HC5 members, respectively, of the $\mathrm{HC}$ group of Bergeat et al. (2001), who noted that no angular size measurements of such group members were available at that time. (As noted already in Section 4.1, these two stars are also identified as members of the unique $\mathrm{CH}$ subgroup of carbon stars.) As such, all Bergeat et al. $T_{\mathrm{EFF}}$ calibrations for $\mathrm{HC}$ stars relied solely on the indirect approaches of SEDs and calibrated color indices.

However, there is an overall good agreement with the CV group temperatures, as seen in Table 6-the only notable discrepancy is for CV6, which in our temperature calibration shows a smoother transition from CV5 than the large $\Delta T=335$ $\mathrm{K}$ drop expected from Bergeat et al. (2001).

\subsection{Reference Angular Diameters}

Using these data, we can refine parameters for the reliable prediction of angular sizes of these stars, as presented in van

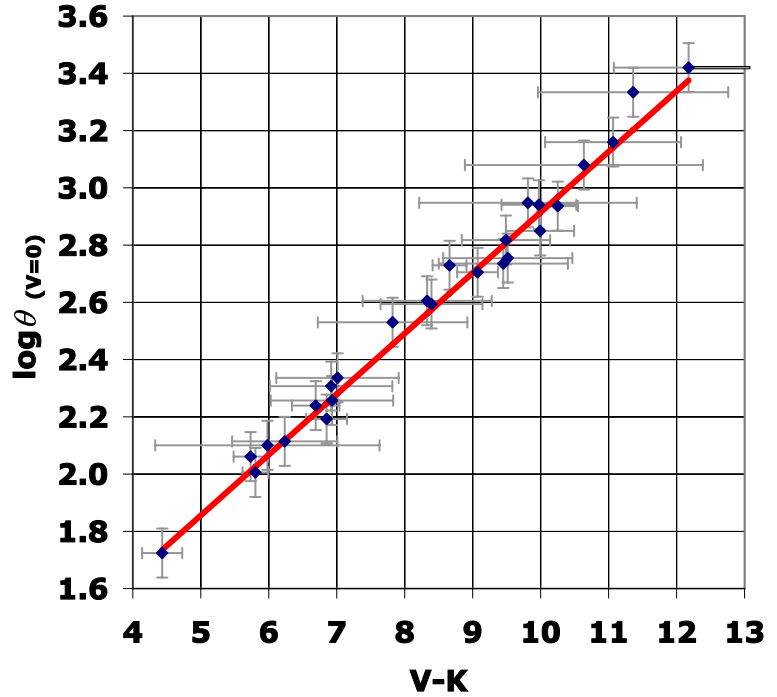

Figure 10. Reference angular sizes $\theta_{V=0}$ vs. $V-K$ color and associated fit (Equation (3)).

(A color version of this figure is available in the online journal.)

Table 6

$T_{\mathrm{EFF}}$ Calibration for the CV Groups of Bergeat et al. (2001)

\begin{tabular}{lrlc}
\hline \hline Group & $N$ & \multicolumn{1}{c}{$\begin{array}{c}T_{\mathrm{EFF}} \\
\text { (This Work) }\end{array}$} & $\begin{array}{c}T_{\mathrm{EFF}} \\
\text { (Bergeat et al. 2001) }\end{array}$ \\
\hline CV2 & 5 & $3145 \pm 315 \mathrm{~K}$ & $3130 \pm 70 \mathrm{~K}$ \\
CV3 & 12 & $2916 \pm 206 \mathrm{~K}$ & $2940 \pm 80 \mathrm{~K}$ \\
CV4 & 5 & $2778 \pm 69 \mathrm{~K}$ & $2790 \pm 130 \mathrm{~K}$ \\
CV5 & 9 & $2638 \pm 154 \mathrm{~K}$ & $2720 \pm 135 \mathrm{~K}$ \\
CV6 & 4 & $2585 \pm 158 \mathrm{~K}$ & $2385 \pm 110 \mathrm{~K}$ \\
\hline
\end{tabular}

Belle (1999). As in that paper, we can use $V$-band data to scale the angular sizes to a common scale, $\theta_{V=0}=\theta \times 10^{V / 5}$, and then fit that reference $\theta_{V=0}$ to the $V-K$ color:

$\log _{10} \theta_{V=0}=(0.796 \pm 0.107)+(0.212 \pm 0.014) \times(V-K)$,

with a rms error of $12 \%$; the $V-K$ uncertainty in this fit is dominated by the $V$ variability (Figure 10). The more general fit to semi-regular variables, Mira variables, and carbon stars in the previous study of van Belle (1999) (which ignored $V-K$ uncertainty) had a slope of $0.218 \pm 0.014$ and an intercept of $0.789 \pm 0.119$ and an rms error of $26 \%$. Overall, these results represent a significant improvement in our ability to predict carbon star angular sizes, solely based upon $V$ and $K$ photometry.

\subsection{Dependency of $T_{E F F}$ on $V_{O}-K_{O}$}

We can examine the effective temperature dependency of our measurements on a commonly utilized color index, $V-K$, particularly in comparison with the synthetic photometry of Aringer et al. (2009). However, to effectively execute this comparison, we have to deredden the observed $V$ and $K$ photometry; fortunately, from the values for $A_{V}$ calculated in Section 3.1, this process is rather straightforward. $A_{K}$ was computed from the standard relationship of $A_{K}=0.108 \times A_{V}$ (Cardelli et al. 1989, assuming $R_{V}=3.1$; other reasonable values for $R_{V}$ do not alter $A_{K}$ in a significant way). A plot of $T_{\mathrm{EFF}}$ versus $V_{0}-K_{0}$ can be seen in Figure 11 . The principal uncertainty in the color is the $V$-band variability, the range of which was taken to be the uncertainty (as was the case in Section 3.1). 


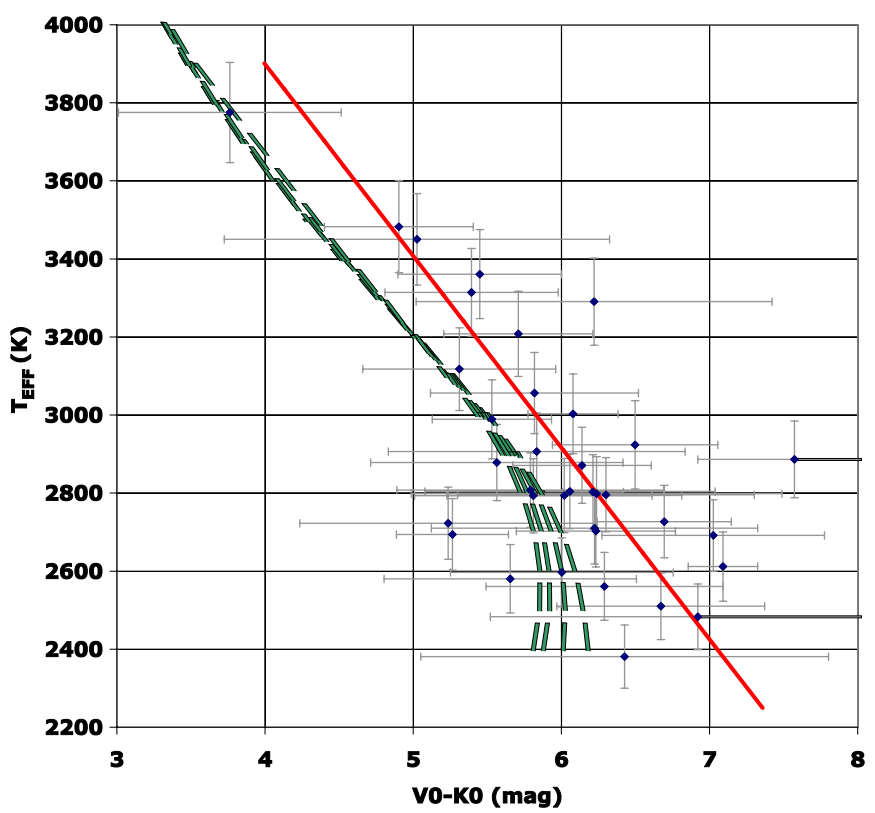

Figure 11. $T_{\mathrm{EFF}}$ as a function of dereddened color $V_{0}-K_{0}$. The red solid line is the linear fit to our data, as discussed in Section 4.4. The green dotted lines are the $\left\{T_{\mathrm{EFF}}, V_{0}-K_{0}\right\}$ tracks from Aringer et al. (2009, e.g., Figure 16) for models with $M=2 M_{\odot}, Z=1 Z_{\odot}, \log (g)=0.0$, and $\mathrm{C} / \mathrm{O}=2.00,1.40$, 1.10 , and 1.05 (left to right).

(A color version of this figure is available in the online journal.)

Referencing a simple linear fit line to the central $V_{0}-K_{0}$ color of 6 , we derived the following fit presented in Figure 11:

$$
T_{\mathrm{EFF}}=\left[\left(V_{0}-K_{0}\right)-6\right] \times(491 \pm 109)-(2917 \pm 53),
$$

with a standard linear fit with errors in both dimensions (Press et al. 1992); the reduced $\chi_{v}^{2}$ was 0.698. Omitting the data point from HD 59643, previously discussed as an outlier in Section 4.2 , alters the slope only slightly to $-525 \pm 141$ with a $\chi_{v}^{2}=0.704$ and does not change the intercept significantly (the other high-velocity carbon star, HD 30443, did not have $V$ data and was not considered in either fit).

Also plotted on Figure 11 are the corresponding model points from Aringer et al. (2009; see Figure 16 of that article, albeit with transposed axes). Selecting the models with $M=2 M_{\odot}, Z=$ $1 Z_{\odot}$, and $\log (g)=0.0$ and a range of carbon abundances, $\mathrm{C} / \mathrm{O}=1.05,1.10,1.40$, and 2.00 , we can compare the predicted dependency of $T_{\mathrm{EFF}}$ on $V_{0}-K_{0}$. Our observations agree on the slope of the relationship in the range of $V_{0}-K_{0}=\{4,5.5\}$, although there is an offset of $\Delta T_{\mathrm{EFF}}=+200 \mathrm{~K}$; redward of $V_{0}-K_{0}>5.5$, the models drop in $T_{\mathrm{EFF}}$ at a constant color of $V_{0}-K_{0} \sim 6$, a feature not captured by our simple linear fit. However, in a qualitative sense, our observational data do exhibit an increasing spread in $V_{0}-K_{0}$ at a given $T_{\mathrm{EFF}}$ for temperatures below $3000 \mathrm{~K}$, which perhaps is indeed indicative of a spread in $\mathrm{C} / \mathrm{O}$, as suggested by the Aringer et al. (2009) synthetic photometry. The blueward shift of the models relative to observations at a given $T_{\mathrm{EFF}}$ was already seen in this previous investigation, which linked this phenomenon to concerns about the applied corrections of interstellar reddening and the limitations of non-simultaneous photometry. The former concern is perhaps mitigated by our treatment of the subject in this investigation; the latter concern remains an area where improvements can be made, both from the specific standpoint of simultaneous measurements of $V$ and $K$ photometry and the general application of $F_{\mathrm{BOL}}$ determinations from (spectro-)

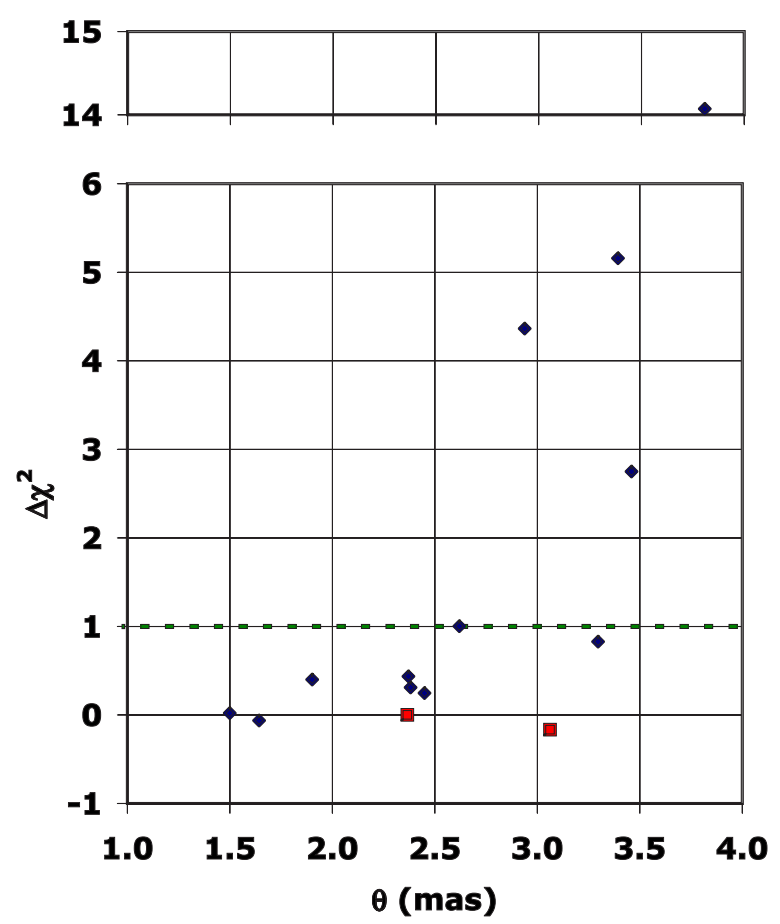

Figure 12. Difference in reduced $\chi_{v}^{2}$ fit values for elliptical vs. circular on-sky photosphere fits; blue diamonds are carbon stars and red squares are the giant check stars.

(A color version of this figure is available in the online journal.)

photometry. Broadly speaking, this subject could also be an area where the limitations of hydrostatic models as applied to even mildly variable objects are highlighted.

\subsection{Departures from Spherical Symmetry}

As shown in Section 2.6.2, a simple UD fit to the visibility data does not show a constant value as a function of on-sky position angle, for those stars for which we have sufficient data. It appears that, regardless of the cause (which will be discussed below), it is possible that all carbon stars examined in sufficient detail this way will exhibit similar departures from circular symmetry.

In this study, "large" stars with $\theta>2.5$ mas observed by the PTI have an average difference between their reduced chisquared values for elliptical versus circular on-sky photospheric fits of $\overline{\Delta \chi_{v}^{2}}=4.70$, indicative of the significant improvement of the elliptical fits over the circular fits (Figure 12). With the exception of IRC +40067 , all of the stars in this range have $\Delta \chi_{v}^{2} \gtrsim 1$ (and typically $\gg 1$ ); as noted in Section 2.6.2, only IRC +40067 has $\Delta \chi_{v}^{2}$ slightly less than one, 0.83 . For "intermediate" sized stars with $1.75<\theta<2.5$ mas, $\overline{\Delta \chi_{v}^{2}}=$ 0.35 and Figure 12 further shows that while the improvement does not rise to the significance level of $\Delta \chi_{v}^{2} \geqslant 1$, it is still $\Delta \chi_{v}^{2}>0$ for all stars in this size range. Only for the two smallest stars, with $\theta<1.75$ mas, is the comparison between circular and elliptical photospheric fits moot, with $\overline{\Delta \chi_{v}^{2}} \simeq 0$ (and comparable to the giants). By comparison, for our giant check stars, $\overline{\Delta \chi_{v}^{2}}=-0.01$, even though one is in the "intermediate" size range and one is in the "large" size range.

One possible explanation for the trend of increasing $\Delta \chi_{v}^{2}$ with increasing $\theta$ is that, for the stars with larger apparent sizes, the data are of sufficiently high signal-to-noise $(\mathrm{S} / \mathrm{N})$ to detect departures from sphericity-departures that are present in essentially all carbon stars but that merely remain undetected 


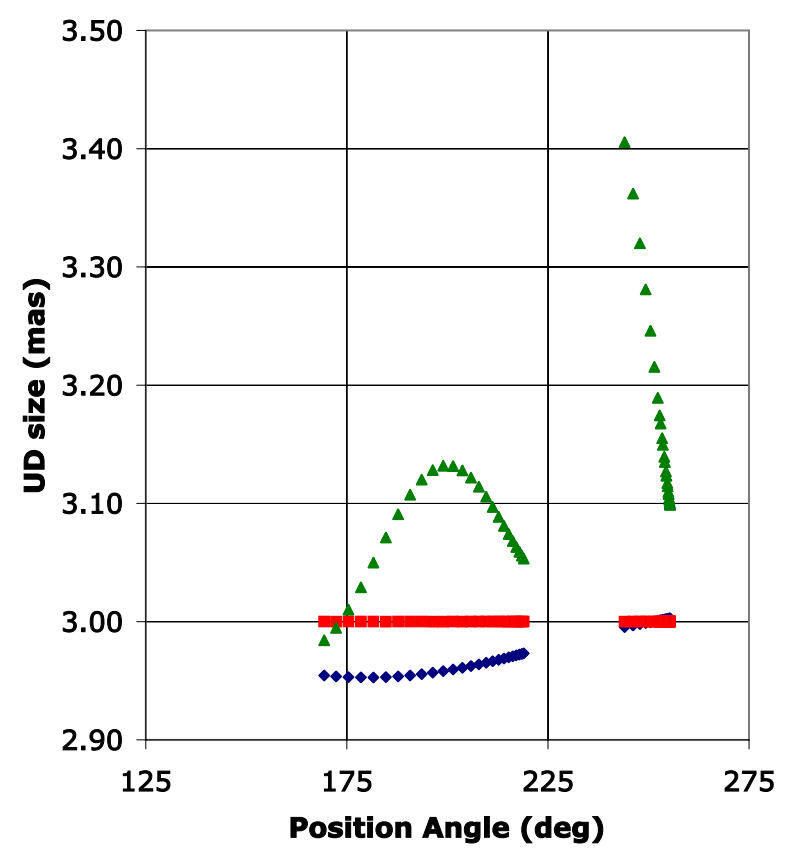

Figure 13. Indicated uniform disk angular size expected from the PTI NS and NW single-baseline observations of three toy models at $\delta=+0^{\circ}$ : (1) a plain disk, 3.0 mas in diameter (red squares), (2) adding a 1.0 mas diameter spot to (1), offset from the disk center by 0.75 mas, with $25 \%$ greater flux emission than the rest of the disk (blue diamonds); (3) adding a circumstellar ring to (1), projected to be $12 \times 2$ mas with an inner hole of $6 \times 1$ mas, and $3 \%$ of the flux of the central star (green triangles). The left-hand grouping of points is consistent with the PTI's $109 \mathrm{~m}$ NS baseline and the right-hand grouping is constant with the PTI's $85 \mathrm{~m}$ SW baseline.

(A color version of this figure is available in the online journal.)

for the smaller stars in our sample. (The proposition that, for these sizes in question, increasing $\theta$ means increasing the $\mathrm{S} / \mathrm{N}$ is considered in detail in the Appendix.) An earlier InfraredOptical Telescope Array study of evolved stars by Ragland et al. (2006) included 10 semi-regular (non-Mira) carbon stars, of which four of these objects indicated detectable asymmetries in their closure phase data. Within the context of $\mathrm{S} / \mathrm{N}$-dependent detections, it is unsurprising that the asymmetric objects are, on average, $\sim 30 \%-50 \%$ larger than those objects for which no asymmetries were detected.

There are three likely scenarios for these observed departures from spherical symmetry: the presence of a pronounced stellar spot (region of increased flux) on a circularly symmetric stellar photosphere; the presence of a disk around a circularly symmetric stellar photosphere; or, a truly elliptical (in appearance) photosphere. We will examine the first two possibilities in Section 4.5.1 and the third in Section 4.5.2.

\subsubsection{Surface Inhomogeneities: Spots, Disks}

When viewed by a single-baseline interferometer such as the PTI, a circular, but non-uniform, stellar photosphere could produce data sets that give on-sky position-angle-dependent UD sizes. This fact is illustrated in Figure 13 with three toy models. The first model is merely a simple UD of 3 mas in diameter, observed by the PTI's NS and NW baselines (as is the case for the real stars in this study), at a declination of $\delta=+0^{\circ}$ for hour angles (HAs) between HA $=\left\{-3^{\mathrm{h}},+3^{\mathrm{h}}\right\}$. Using NExScI's Visibility Modeling Tool (VMT), ${ }^{11}$ a simple set of $V^{2}$ values were synthesized and UD values were fit to the

\footnotetext{
11 http://nexsciweb.ipac.caltech.edu/vmt/vmtWeb/
}

individual $V^{2}$ points. For the simple UD, each $V^{2}$ (HA, baseline) point produced an apparent size of 3 mas.

To illustrate the case of a stellar hot spot, we added a 1 mas spot in our VMT toy model, which had $25 \%$ greater flux emitted per unit area than the rest of the star, offset from the center of the toy model by 0.75 mas. A flux differential of this magnitude corresponds to a $\Delta T$ of $\sim 175 \mathrm{~K}$ for our $\sim 3000 \mathrm{~K}$ stars. As seen in Figure 13, this procedure introduces a gentle variation of $2 \%$ in apparent UD diameter, from 2.95 to 3.0 mas, as the on-sky position angle varies with HA and baseline.

A second iteration to our toy model was to add not a spot but a circumstellar disk, projected such that its outer dimensions were $12 \times 2$ mas on-sky, with inner dimensions of $6 \times 1$ mas, with a major axis position angle of $45^{\circ}$. What is striking about the disk toy model-and this fact is representative of all other disk toy models we tried, at a variety of inclinations-is that while there is a gentle variation in fitted UD sizes for $V^{2}$ data from a given baseline, there is a strong discontinuity in UD sizes between baselines. This result is due to the combination of differing projection lengths for the two PTI baselines on a complex overresolved structure, such as a disk. In general, any putative disk-like structures that surround these objects will be greater in angular extent than the host stars. Since the host stars are in the "sweet spot" of angular resolution for the PTI (2-3 mas), which corresponds to the mid-point of the central lobe of the visibility function, structures that are $\gtrsim 1.5 \times$ greater in size will be overresolved and the visibility contribution will be nearly zero. While it is perhaps possible that a specific set of parameters for disk toy models could result in "smoother" variation in UD sizes in those specific cases, the complete lack of such discontinuities found in our observational data set lead us to conclude that disks are an unlikely explanation for our observations.

Additionally, such a dusty disk would need to be optically thick even in the $K$ band (e.g., see the illustration in Figure 13(b) of Johnson \& Jones (1991) and the related discussion in Section 5 of that paper.) However, our expectation is that such a dense dust disk is inconsistent with the overall reddening levels for these stars-our SED results of $A_{V} \simeq 0.75-2.50$ (Section 3.1) imply $A_{K} \simeq 0.08-0.28$, of which most if not all of this effect at these distances should be interstellar and not circumstellar.

Certainly for objects represented by the extreme case of IRC +10216 , with $15 \%-24 \%$ polarization features in the $H$ band and a mass-loss rate of $\sim 2 \times 10^{-5} M_{\odot} \mathrm{yr}^{-1}$ (Murakawa et al. 2005), such considerations cannot be discounted. However, for the carbon stars in our sample, the polarization levels are closer to the 1\% level (López \& Hiriart 2011a, 2011b) and the massloss rates are $<10^{-6.25} M_{\odot} \mathrm{yr}^{-1}$.

However, the possibility of significant hot spots remains quite likely. This hypothesis seems specifically supported by stellar models: Freytag \& Höfner (2008) find that spatial inhomogeneities, induced by huge convection cells, should be significant surface features on AGB stars. Chiavassa et al. (2010, 2011b) find that for red supergiants such as Betelgeuse, significant photocentric excursions (up to $\sim 7.5 \%$ of the stellar radius) are expected in the visible due to smaller-scale convection cells; in the $H$ band, Chiavassa et al. (2011c) show that models have larger "conspicuous spots" due to subphotospheric convective cells, which have lives of several years.

These sorts of structures - if present in the related but nonidentical carbon stars - could lead to the "hot spot" observable we have treated here with our toy model. A specific numerical solution is not provided for each of the stars in our sample 
because the parameters for even a single simple hot spot (e.g., size, location, $\Delta T$ ) are too complex (and therefore, degenerate) to be uniquely solvable with our data.

\subsubsection{Oblateness and Mass Loss}

A plain spheroid of gas will deform into an oblate spheroid when subjected to rotation, which in turn will present itself upon the sky (roughly) as an ellipsoidal photosphere. Furthermore, oblateness due to rotation may be intrinsically connected to the phenomenon of mass loss for carbon stars. The previous studies of Kahn \& West (1985) and Johnson \& Jones (1991) established that mass loss for these stars must be aspherical, without positing the underlying cause. The latter study based its empirical result on polarization observations, which have been expanded upon in López \& Hiriart (2011a, 2011b). Much of the attention on carbon star mass loss has concentrated on chemistry and dust grain size (e.g., Mattsson \& Höfner 2011). However, there has been at least some discussion in the literature exploring the relationship between rotation and mass-loss rates-e.g., Dorfi \& Höfner (1996) discuss non-spherical winds due to "slowly" rotating C-rich AGB stars, up to $v \sin i \sim 7 \mathrm{~km} \mathrm{~s}^{-1}$.

For the carbon star V Hya, a spectroscopic rotation velocity of $v \sin i=13.5 \mathrm{~km} \mathrm{~s}^{-1}$ is acknowledged as "rapid" by Barnbaum et al. (1995), who note in their conclusions that the star would "not be spherically symmetric." Such rotation is consistent with a large fraction $(>50 \%)$ of planetary nebulae being aspherical (Zuckerman \& Aller 1986); this fact is potentially connected to central star rotation (Mufson \& Liszt 1975; Phillips \& Reay 1977; Pascoli 1987).

Following Equation (A1) of van Belle et al. (2006), the range of oblatenesses found in Section 2.6.2 roughly corresponds (for $\bar{R} \sim 360 R_{\odot}$, from Section 3.3) to $v \sin i$ values of $9-17 \mathrm{~km} \mathrm{~s}^{-1}$ for $\bar{M} \sim 2 M_{\odot}$ and $7-13.5 \mathrm{~km} \mathrm{~s}^{-1}$ for $\bar{M} \sim 1.2 M_{\odot}$. This range of putative $v \sin i$ values actually bridges the region between the somewhat qualitatively defined regimes of "slow" rotation as defined by Dorfi \& Höfner (1996) and the "rapid" rotation of Barnbaum et al. (1995). The values exceed the macroturbulent velocities reported by Lambert et al. (1986) (4 $\lesssim V_{\text {macro }} \lesssim 7 \mathrm{kms}^{-1}$ ), but we are cautious about being too concerned with this conflict, noting that that investigation relied upon plane-parallel models; their assertion that the effects of spherical extension were found to be unimportant based upon the method of Nordlund (1984) has been contradicted somewhat in subsequent studies. For example, Aringer et al. (2009) show $\sim 20 \%$ reductions in $\mathrm{SiO}$ equivalent widths at a given $T_{\mathrm{EFF}}$ when plane-parallel model atmospheres are replaced by spherical model atmospheres.

We examined the stars for which we could derive oblate fits in Section 2.6.2 against the mass-loss data of Claussen et al. (1987). Other mass-loss references were examined (Schöier \& Olofsson 2001; Groenewegen et al. 2002; Guandalini et al. 2006) but insufficient (if any) overlap was found with our carbon sample. A statistically weighted fit of oblateness $\left(o_{a b}=a / b-1\right)$ for those stars in our sample with robust, or even marginal, $o_{a b}$ data versus the mass-loss rates of Claussen et al. (1987) gives

$$
o_{a b}=(0.11 \pm 0.06) \times \log _{10}\left(\frac{d M}{d t}\right)+(0.82 \pm 0.41)
$$

with a reduced chi-squared of $\chi_{v}^{2}=0.49$, as can be seen in Figure 14. This result is not statistically significant-a flat line of intercept 0.12 has $\chi_{v}^{2}=0.99$, so the level of improvement is not

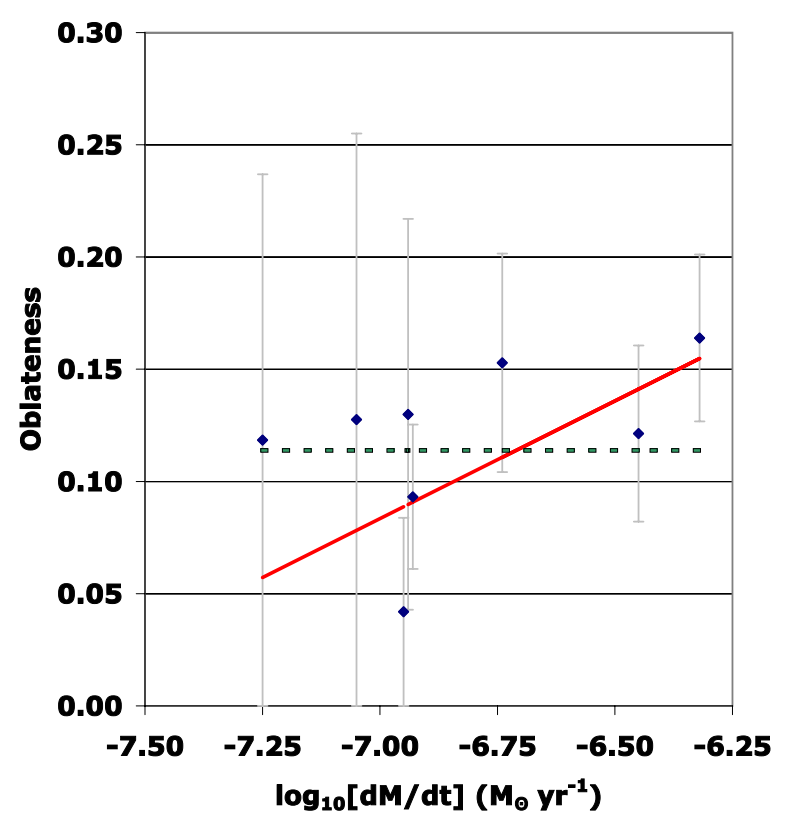

Figure 14. Our values for oblateness $o_{a b}=a / b-1$ vs. mass-loss rates from Claussen et al. (1987), with a solid fit line described in Equation (5) with $\chi_{v}^{2}=0.49$; a dotted flat line is also shown fit to the data, with intercept an $o_{a b}=0.12$ and $\chi_{v}^{2}=0.99$.

(A color version of this figure is available in the online journal.)

quite $\Delta \chi_{v}^{2}=1$-but is a tantalizing suggestion that oblateness is a significant factor when considering carbon star mass-loss rates.

Spectral measurements of carbon star rotational velocities are challenging, given the complicated spectra of these objects that are characterized principally by a dense set of vibrationrotation transitions of $\mathrm{CN}$ and $\mathrm{C}_{2}$ (Barnbaum et al. 1995); direct measurements of equivalent widths are not possible without using spectral synthesis models.

Although Pereira \& Roig (2006) characterize rapid rotation among AGB stars as "very rare," this statement was made without substantiation. Indeed, de Medeiros (2004) point out that in their evolved star rotation catalog (de Medeiros \& Mayor 1999), "a growing list" of evolved stars present moderate to fast rotation: 200 of the 1540 non-carbon FGK evolved stars in their catalog have $v \sin i>10 \mathrm{~km} \mathrm{~s}^{-1}$, although the investigation of Carlberg et al. (2011b) specifically directed at characterizing the frequency of $\mathrm{K}$ giant rapid rotation (again, $v \sin i>10 \mathrm{~km} \mathrm{~s}^{-1}$ ) recovered a frequency of $2.2 \%$. It simply may be the case that, for carbon stars, rotation rates have not been comprehensively measured due to the difficulty of the task.

For the oblateness explanation, the parent population could plausibly be evolved rapid rotators, or stars spun up from exoplanet ingestion (Soker \& Harpaz 2000; Soker 2001; Carlberg et al. 2009, 2011a). Indeed, Soker (2001) argues that fairly small exoplanets $\left(M \sim 0.01-0.1 M_{\text {Jupiter }}\right)$ within 2 AU could be sufficient to spin up stars as they reach the AGB. If correct, this hypothesis is significantly boosted by recent Kepler results that indicate $\sim 23 \%$ of FGK stars have 1-3 $M_{\text {Earth }}\left(0.003-0.01 M_{\text {Jupiter }}\right)$ planets within $0.25 \mathrm{AU}$ (Fressin et al. 2013). That result is further extended by indications that the number of planets as a function of orbital period ( $\delta N_{\text {planets }} / \delta \log P$ ) remains constant (Dong \& Zhu 2012) out to 500 days, which implies $\sim 45 \%$ of FGK stars have 1-3 $M_{\text {Earth }}$ planets out to $1.2 \mathrm{AU}$ (with additional planets yet to be found in the range of 1.2-2.0 AU). 

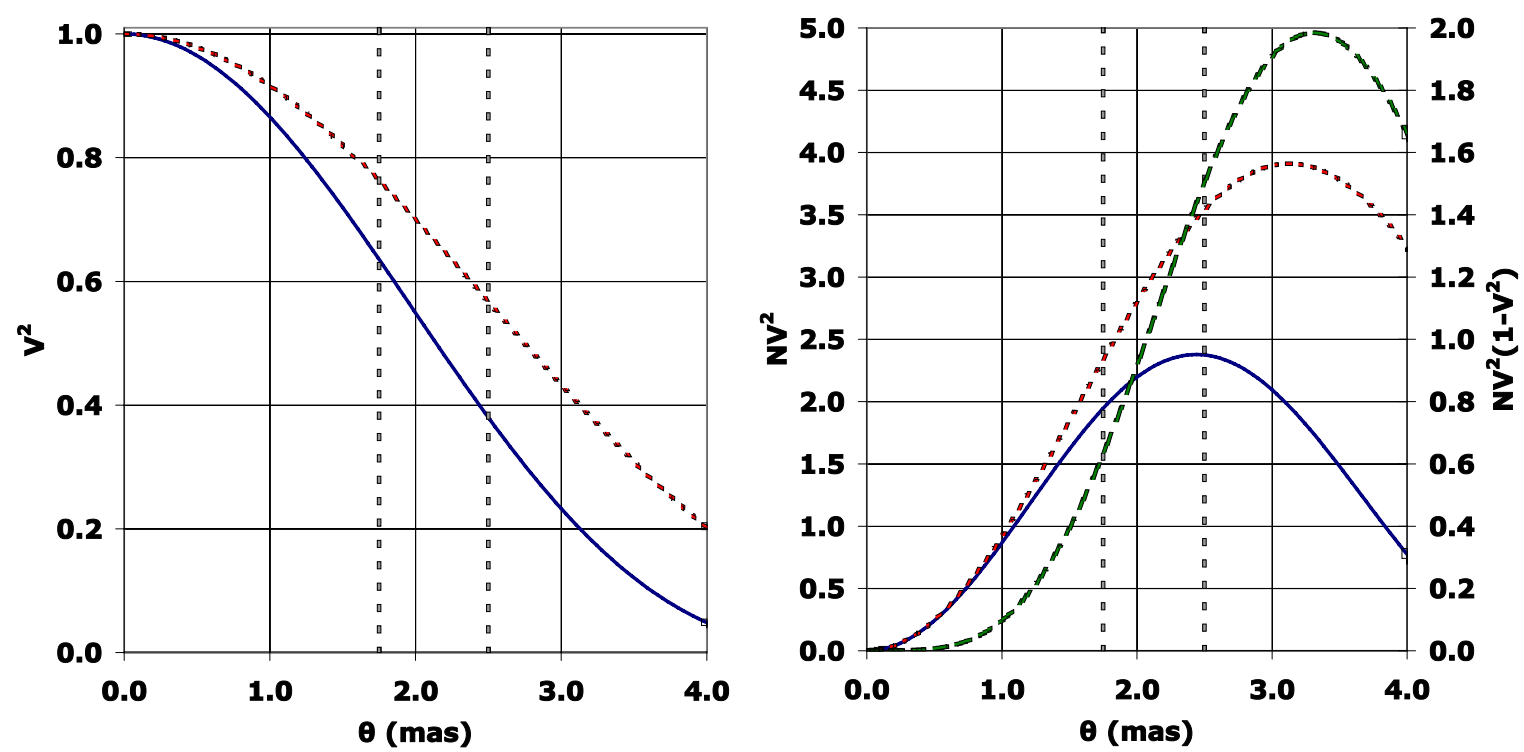

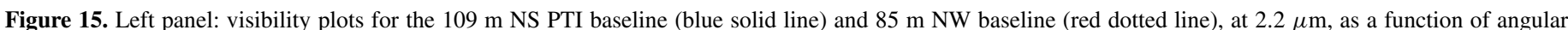

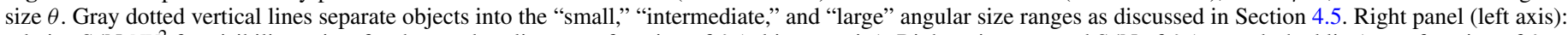

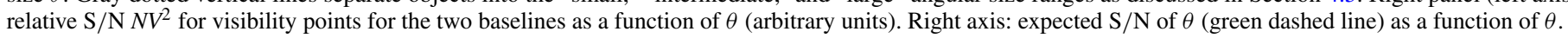

(A color version of this figure is available in the online journal.)

\section{CONCLUSIONS}

Angular size measurements are presented for a homogenous data set of 41 carbon stars, the largest such single study to date. Our results compare favorably with the previous studies of Dyck et al. (1996b) and Bergeat et al. (2001, 2002a, $2002 \mathrm{~b}$ ) and provide new calibrations of $T_{\mathrm{EFF}}$ versus Yamashita spectral type and carbon star angular predictions. As a group, these stars have a median $T_{\mathrm{EFF}}=2800 \pm 270 \mathrm{~K}$, although this temperature shows a decrease with increasing Yamashita spectral type. Notably, our data indicate that some carbon stars clearly present non-spherical solutions, due to either hot spots or rotational oblateness, and suggest that potentially all carbon stars will present such solutions when examined in sufficient detail.

Follow-up observations have the potential to separate the hypotheses of hot spots versus rotational oblateness, in two ways. First, collecting data on the current set of stars with similar detail will present a long time baseline ( $\sim$ years) between observations, and presumably any putative hot spots will migrate during that interval. If each carbon star presents repeatable degrees of oblateness and position angles upon the sky, the notion of hot spots due to stochastically varying convection seems less likely. Second, increasing the sample size, particularly as it can populate Figure 14 to the point of statistical significance of the $o_{a b}$ versus mass loss relationship, would increase the confidence in or motivate discarding the rotation hypothesis.

Unfortunately, continuing this investigation with the PTI is not possible, since the facility was decommissioned in 2009. However, newer interferometric imaging facilities such as the CHARA Array and the VLTI, particularly with their near-IR to mid-IR multi-baseline instruments such as MIRC, PIONIER, and (in the future) MATISSE and GRAVITY, have the potential to carry on this line of inquiry. If non-spherical surface morphology is indeed a general characteristic of carbon stars, the nature of that morphology as it relates to the parameters of the main-sequence parent population, the overall angular momentum history (including possible exoplanet ingestion), and the structure of evolved stellar atmospheres become quite interesting to consider.

We thank the staff of the Palomar Observatory-in particular, Kevin Rykoski-for support during our observations and the PTI Collaboration for a generous allotment of time that made this research possible. We have made extensive use of the SIMBAD database and the VizieR catalog access tool, operated by the CDS in Strasbourg, France (Ochsenbein et al. 2000). This research has made use of the AFOEV database, operated at CDS, France. This research has made use of NASA's Astrophysics Data System. Funding for the PTI was provided to the Jet Propulsion Laboratory under its TOPS (Towards Other Planetary Systems), ASEPS (Astronomical Studies of Extrasolar Planetary Systems), and Origins programs and from the JPL Director's Discretionary Fund. Portions of this work were performed at the Jet Propulsion Laboratory, California Institute of Technology under contract with the National Aeronautics and Space Administration.

G.v.B. would like to thank H. M. Dyck for the original inspiration and direction to make interferometric observations of both carbon stars and rotationally oblate stars. G.v.B. also thanks R. Canterna for generous support during the (very) early phases of this work. Helpful input for this article resulted from discussions with and feedback from Travis Barman, Kaspar von Braun, Jay Farihi, Terry Jones, Phil Massey, and Greg Sloan. Portions of this research were conducted while G.v.B. was in residence at the European Southern Observatory; funding for this research for G.v.B. has been generously provided in part by Lowell Observatory. This material is based upon work supported by the National Science Foundation under Grant No. AST1212203 and NASA Grant NNX13AF01G. C.P., B.A., and J.H. were supported by the Projects P23006 of the Austrian Science Fund (FWF). The research leading to these results has received funding from the European Community's Seventh Framework Programme under Grant Agreement 226604. 


\section{APPENDIX}

In Section 4.5, we suggest that, for our carbon star sample, data quality improves directly with increasing $\theta$. This improvement in data quality is illustrated qualitatively in Figure 15. In the left panel, notional $2.2 \mu \mathrm{m}$ visibility curves for both the $109 \mathrm{~m} \mathrm{NS}$ and $85 \mathrm{~m}$ NW PTI baselines are shown, with the three size ranges partitioned off with gray dotted vertical lines. In the right panel, we illustrate the relative $\mathrm{S} / \mathrm{N}$ for a fringe-tracking interferometer such as the PTI, which scales as the flux and the visibility squared, $N V^{2}$ (Colavita 1999). Since all of these stars are fairly similar in temperature (Section 3), we shall assume that the flux $N$ simply scales with apparent angular size $\theta$, $N \propto \theta^{2}$. As such, $N V^{2}$ increases rapidly with $\theta$ initially-until the visibility has dropped sufficiently low that these $\mathrm{S} / \mathrm{N}$ curves turn over and drop.

For measurements of angular sizes, however, one may consider as a primary measurement influence not the quantity $V^{2}$, but the distance from unit visibility $\left(1-V^{2}\right)$. Consider how a solution for the visibility curve-and the resulting measure of $\theta$-is constrained by two visibility points of differing amplitudes but identical uncertainties. The low-level point will robustly fix the curve in place, while the high-level point has a large family of visibility curve solutions that fit through it. As such, we have plotted the proxy product of visibility point $\mathrm{S} / \mathrm{N} N V^{2}$ and angular size "goodness" $\left(1-V^{2}\right)$, averaged for both baselines, on the right-hand vertical axis of Figure 15 (right-hand panel). Using the three size regimes of Section 4.5, the angular size regimes are partitioned on the plot. Using this proxy, we can see how angular sizes in the "large" range have roughly $\sim 2 \times$ the $S / N$ of those in the "intermediate" range and $\sim 4 \times$ the $S / N$ of those in the "small" range; the "intermediate" points have $>2 \times$ the $S / N$ of the "small" points.

\section{REFERENCES}

Alksnis, A., Balklavs, A., Dzervitis, U., et al. 2001, BaltA, 10, 1

Aringer, B., Girardi, L., Nowotny, W., Marigo, P., \& Lederer, M. T. 2009, A\&A, 503, 913

Barnbaum, C., Morris, M., \& Kahane, C. 1995, ApJ, 450, 862

Barnbaum, C., Stone, R. P. S., \& Keenan, P. C. 1996, ApJS, 105, 419

Bergeat, J., Knapik, A., \& Rutily, B. 1999, A\&A, 342, 773

Bergeat, J., Knapik, A., \& Rutily, B. 2001, A\&A, 369, 178

Bergeat, J., Knapik, A., \& Rutily, B. 2002a, A\&A, 390, 967

Bergeat, J., Knapik, A., \& Rutily, B. 2002b, A\&A, 390, 987

Bergeat, J., \& Lunel, M. 1980, A\&A, 87, 139

Bergeat, J., Sibille, F., Lunel, M., \& Lefevre, J. 1976, A\&A, 52, 227

Boden, A. F., Colavita, M. M., van Belle, G. T., \& Shao, M. 1998, Proc. SPIE, 3350,872

Boden, A. F., Koresko, C. D., van Belle, G. T., et al. 1999, ApJ, 515, 356

Cardelli, J. A., Clayton, G. C., \& Mathis, J. S. 1989, ApJ, 345, 245

Carlberg, J. K., Majewski, S. R., \& Arras, P. 2009, ApJ, 700, 832

Carlberg, J. K., Majewski, S. R., Arras, P., et al. 2011a, in AIP Conf. Proc. 1331, Planetary Systems Beyond the Main Sequence, ed. S. Schuh, H. Drechsel, \& U. Heber (Melville, NY: AIP), 33

Carlberg, J. K., Majewski, S. R., Patterson, R. J., et al. 2011b, ApJ, 732, 39

Chiavassa, A., Freytag, B., Masseron, T., \& Plez, B. 2011a, A\&A, 535, A22

Chiavassa, A., Pasquato, E., Jorissen, A., et al. 2010, in SF2A-2010: Proceedings of the Annual Meeting of the French Society of Astronomy and Astrophysics, ed. S. Boissier, M. Heydari-Malayeri, R. Samadi, \& D. Valls-Gabaud (Paris: Société Française d'Astronomie et d'Astrophysique), 339

Chiavassa, A., Pasquato, E., Jorissen, A., et al. 2011b, in ASP Conf. Ser. 445, Why Galaxies Care about AGB Stars II: Shining Examples and Common Inhabitants, ed. F. Kerschbaum, T. Lebzelter, \& R. F. Wing (San Francisco, CA: ASP), 169

Chiavassa, A., Pasquato, E., Jorissen, A., et al. 2011c, A\&A, 528, A120

Claussen, M. J., Kleinmann, S. G., Joyce, R. R., \& Jura, M. 1987, ApJS, 65,385

Cohen, J. G., Persson, S. E., Elias, J. H., \& Frogel, J. A. 1981, ApJ, 249, 481

Cohen, M. 1979, MNRAS, 186, 837
Colavita, M. M. 1999, PASP, 111, 111

Colavita, M. M., Wallace, J. K., Hines, B. E., et al. 1999, ApJ, 510, 505

Cox, A. N. (ed.) 2000, in Allen's Astrophysical Quantities (4th ed.; New York: AIP)

Cutri, R. M., Skrutskie, M. F., van Dyk, S., et al. 2003, 2MASS All Sky Catalog of Point Sources, NASA/IPAC Infrared Science Archive, (http://irsa.ipac.caltech.edu/applications/Gator/)

de Medeiros, J. R., \& Mayor, M. 1999, A\&AS, 139, 433

de Medeiros, J. R. 2004, in IAU Symp. 215, Stellar Rotation, ed. A. Maeder \& P. Eenens (San Francisco, CA: ASP), 144

Dong, S., \& Zhu, Z. 2012, arXiv:1212.4853

Dorfi, E. A., \& Höfner, S. 1996, A\&A, 313, 605

Droege, F., T., Richmond, W., M., \& Sallman, M. 2007, yCat, 2271, 0

Dyck, H. M., Benson, J. A., van Belle, G. T., \& Ridgway, S. T. 1996a, AJ, 111,1705

Dyck, H. M., van Belle, G. T., \& Benson, J. A. 1996b, AJ, 112, 294

Eggen, O. J. 1963, AJ, 68, 483

Eggen, O. J. 1972, ApJ, 175, 787

Fressin, F., Torres, G., Charbonneau, D., et al. 2013, ApJ, 766, 81

Freytag, B., \& Höfner, S. 2008, A\&A, 483, 571

Frogel, J. A., Persson, S. E., \& Cohen, J. G. 1980, ApJ, 239, 495

Fukugita, M., Shimasaku, K., \& Ichikawa, T. 1995, PASP, 107, 945

Goebel, J. H., Bregman, J. D., Witteborn, F. C., Taylor, B. J., \& Willner, S. P. 1981, ApJ, 246, 455

Gontcharov, G. A. 2012, AstL, 38, 87

Gottlieb, D. M., \& Upson, W. L., II. 1969, ApJ, 157, 611

Gray, R. O., Corbally, C. J., Garrison, R. F., McFadden, M. T., \& Robinson, P. E. 2003, AJ, 126, 2048

Groenewegen, M. A. T., Sevenster, M., Spoon, H. W. W., \& Pérez, I. 2002, A\&A, 390,511

Guandalini, R., Busso, M., Ciprini, S., Silvestro, G., \& Persi, P. 2006, A\&A, 445,1069

Herwig, F. 2005, ARA\&A, 43, 435

Iben, I., Jr., \& Renzini, A. 1983, ARA\&A, 21, 271

Johnson, H. L. 1968, in Interstellar Extinction, ed. B. M. Middlehurst \& L. H. Aller (Chicage, IL: Univ. Chicago Press), 167

Johnson, J. J., \& Jones, T. J. 1991, AJ, 101, 1735

Jørgensen, U. G., Hron, J., \& Loidl, R. 2000, A\&A, 356, 253

Joyce, R. R. 1998, AJ, 115, 2059

Kahn, F. D., \& West, K. A. 1985, MNRAS, 212, 837

Keenan, P. C. 1993, PASP, 105, 905

Keenan, P. C. 1994, in ASP Conf. Ser. 60, The MK Process at 50 Years: A Powerful Tool for Astrophysical Insight, ed. C. J. Corbally, R. O. Gray, \& R. F. Garrison (San Francisco, CA: ASP), 15

Keenan, P. C., \& McNeil, R. C. 1989, ApJS, 71, 245

Keenan, P. C., \& Morgan, W. W. 1941, ApJ, 94, 501

Knapik, A., \& Bergeat, J. 1997, A\&A, 321, 236

Kurucz, R. L., \& Avrett, E. H. 1981, SAOSR, 391

Lambert, D. L., Gustafsson, B., Eriksson, K., \& Hinkle, K. H. 1986, ApJS, 62,373

Lançon, A., \& Wood, P. R. 2000, A\&AS, 146, 217

Loidl, R., Lançon, A., \& Jørgensen, U. G. 2001, A\&A, 371, 1065

Lomb, N. R. 1976, Ap\&SS, 39, 447

López, J. M., \& Hiriart, D. 2011a, AJ, 142, 11

López, J. M., \& Hiriart, D. 2011b, RMxAA, 47, 63

Lynga, G. 1982, A\&A, 109, 213

Mattsson, L., \& Höfner, S. 2011, A\&A, 533, A42

Mendoza, V., E. E., \& Johnson, H. L. 1965, ApJ, 141, 161

Milne, D. K., \& Aller, L. H. 1980, AJ, 85, 17

Moreno, H. 1971, A\&A, 12, 442

Mozurkewich, D., Johnston, K. J., Simon, R. S., et al. 1991, AJ, 101, 2207

Mufson, S. L., \& Liszt, H. S. 1975, ApJ, 202, 183

Murakawa, K., Suto, H., Oya, S., et al. 2005, A\&A, 436, 601

Neugebauer, G., \& Leighton, R. B. 1969, Two-micron Sky Survey. A Preliminary Catalogue (Washington, DC: NASA)

Noguchi, K., Kawara, K., Kobayashi, Y., et al. 1981, PASJ, 33, 373

Nordlund, A. 1984, in Iterative Solution of Radiative Transfer Problems with Spherical Symmetry Using a Single-ray Approximation, ed. W. Kalkofen (Cambridge: Cambridge Univ. Press), 211

Ochsenbein, F., Bauer, P., \& Marcout, J. 2000, A\&AS, 143, 23

Ohnaka, K., \& Tsuji, T. 1996, A\&A, 310, 933

Ohnaka, K., \& Tsuji, T. 1999, A\&A, 345, 233

Paladini, C., Aringer, B., Hron, J., et al. 2009, A\&A, 501, 1073

Paladini, C., van Belle, G. T., Aringer, B., et al. 2011, A\&A, 533, A27

Pascoli, G. 1987, A\&A, 180, 191

Pereira, C. B., \& Roig, F. 2006, A\&A, 452, 571

Phillips, J. P., \& Reay, N. K. 1977, A\&A, 59, 91 
Piirola, V. 1976, HelR, 1, 0

Press, W. H., Teukolsky, S. A., Vetterling, W. T., \& Flannery, B. P. 1992, in Numerical Recipes in C. The Art of Scientific Computing (2nd ed.; Cambridge: Cambridge Univ. Press)

Ragland, S., Traub, W. A., Berger, J.-P., et al. 2006, ApJ, 652, 650

Rowan-Robinson, M., \& Harris, S. 1983, MNRAS, 202, 797

Rufener, F. 1976, A\&AS, 26, 275

Samus, N. N., Durlevich, O. V., et al. 2009, yCat, 1, 2025

Scargle, J. D. 1982, ApJ, 263, 835

Schöier, F. L., \& Olofsson, H. 2001, A\&A, 368, 969

Scholz, M., \& Takeda, Y. 1987, A\&A, 186, 200

Skiff, B. A. 2010, yCat, 1, 2023

Smith, B. J., Price, S. D., \& Baker, R. I. 2004, ApJS, 154, 673

Sneden, C. 1973, ApJ, 184, 839

Soker, N. 2001, MNRAS, 324, 699

Soker, N., \& Harpaz, A. 2000, MNRAS, 317, 861

Straizys, V., Kazlauskas, A., Jodinskiene, E., \& Bartkevicius, A. 1989, BICDS, 37,179

Tanaka, M., Letip, A., Nishimaki, Y., et al. 2007, PASJ, 59, 939

Thompson, R. R., Creech-Eakman, M. J., \& van Belle, G. T. 2002, ApJ, 577,447

Thronson, H. A., Jr., Latter, W. B., Black, J. H., Bally, J., \& Hacking, P. 1987, ApJ, 322, 770

Tsuji, T. 1981, JApA, 2, 95

Tsuji, T., Tomioka, K., Sato, H., Iye, M., \& Okada, T. 1991, A\&A, 252, L1

Tuthill, P. G., Monnier, J. D., \& Danchi, W. C. 2005, ApJ, 624, 352 van Belle, G. T. 1999, PASP, 111, 1515

van Belle, G. T. 2012, A\&ARv, 20, 51

van Belle, G. T., Ciardi, D. R., ten Brummelaar, T., et al. 2006, ApJ, 637, 494

van Belle, G. T., Ciardi, D. R., Thompson, R. R., Akeson, R. L., \& Lada, E. A. 2001, ApJ, 559, 1155

van Belle, G. T., Creech-Eakman, M. J., \& Hart, A. 2009, MNRAS, 394, 1925

van Belle, G. T., Lane, B. F., Thompson, R. R., et al. 1999, AJ, 117, 521

van Belle, G. T., \& Thompson, R. R. 1999, BAAS, 31, 1435

van Belle, G. T., Thompson, R. R., \& Creech-Eakman, M. J. 2002, AJ, 124,1706

van Belle, G. T., \& van Belle, G. 2005, PASP, 117, 1263

van Belle, G. T., van Belle, G., Creech-Eakman, M. J., et al. 2008, ApJS, 176,276

van Belle, G. T., \& von Braun, K. 2009, ApJ, 694, 1085

Watson, C., Henden, A. A., \& Price, A. 2012, yCat, 1, 2027

Watson, C. L. 2006, SASS, 25, 47

Whitelock, P. A., Feast, M. W., Marang, F., \& Groenewegen, M. A. T. 2006, MNRAS, 369, 751

Yamashita, Y. 1972, AnTok, 13, 169

Yamashita, Y. 1975a, PASJ, 27, 325

Yamashita, Y. 1975b, AnTok, 15, 47

Zamora, O., Abia, C., Plez, B., Domínguez, I., \& Cristallo, S. 2009, A\&A, 508, 909

Zdanavicius, K., Nikonov, V. B., Sudzius, J., et al. 1972, VilOB, 34, 3

Zuckerman, B., \& Aller, L. H. 1986, ApJ, 301, 772 